\title{
Sectional convexity of epigraphs of conjugate mappings with applications to robust vector duality
}

\author{
N. Dinh* $\quad$ D.H. Long ${ }^{\dagger}$
}

October 24, 2019

Dedicated to Professor Hoang Tuy's $90^{\text {th }}$ birthday

\begin{abstract}
This paper concerns the robust vector problems

(RVP) WMin $\left\{F(x): x \in C, G_{u}(x) \in-S, \forall u \in \mathcal{U}\right\}$,
\end{abstract}

where $X, Y, Z$ are locally convex Hausdorff topological vector spaces, $K$ is a closed and convex cone in $Y$ with nonempty interior, and $S$ is a closed, convex cone in $Z, \mathcal{U}$ is an uncertainty set, $F: X \rightarrow Y^{\bullet}, G_{u}: X \rightarrow Z^{\bullet}$ are proper mappings for all $u \in \mathcal{U}$, and $\emptyset \neq C \subset X$. Let $A:=C \cap\left(\bigcap_{u \in \mathcal{U}} G_{u}^{-1}(-S)\right)$ and $I_{A}: X \rightarrow Y^{\bullet}$ be the indicator map defined by $I_{A}(x)=0_{Y}$ if $x \in A$ and $I_{A}(x)=+\infty_{Y}$ if $x \notin A$.

It is well-known that the epigraph of the conjugate mapping $\left(F+I_{A}\right)^{*}$, in general, is not a convex set. We show that, however, it is " $k$-sectionally convex" in the sense that each section form by the intersection of epi $\left(F+I_{A}\right)^{*}$ and any translation of a "specific $k$-direction-subspace" is a convex subset, for any $k$ taking from int $K$.

The key results of the paper are the representations of the epigraph of the conjugate mapping $\left(F+I_{A}\right)^{*}$ via the closure of the $k$-sectionally convex hull of a union of epigraphs of conjugate mappings of mappings from a family involving the data of the problem (RVP). The results are then given rise to stable robust vector/convex vector Farkas lemmas which, in turn, are used to establish new results on robust strong stable duality results for (RVP). It is shown at the end of the paper that, when specifying the result to some concrete classes of scalar robust problems (i.e., when $Y=\mathbb{R}$ ), our results cover and extend several corresponding known ones in the literature.

Key word: Robust vector optimization, robust convex optimization, robust convex strong duality, robust stable vector Farkas lemma, sectionally convex sets, sectionally closed sets.

Mathematics Subject Classification: 90C25, 49N15, 90C31

*International University, Vietnam National University - HCMC, Linh Trung ward, Thu Duc district, Ho Chi Minh city, Vietnam (ndinh@hcmiu.edu.vn). Parts of the work of this author is supported by the NAFOSTED, Vietnam.

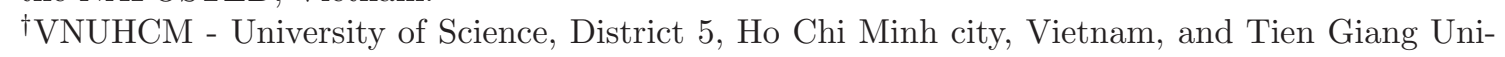
versity, Tien Giang town, Vietnam (danghailong@tgu.edu.vn). 


\section{Introduction}

Let $X, Y, Z$ be locally convex Hausdorff topological vector spaces (briefly, lcHtvs) with topological dual spaces denoted by $X^{*}, Y^{*}, Z^{*}$, respectively. The only topology we consider on dual spaces is the weak*-topology. For a set $U \subset X$, we denote by $\operatorname{cl} U$, int $U$, co $U$, and clco $U$ the closure, the interior, the convex hull, and the closed and convex hull of $U$, respectively. Note that $\operatorname{cl} \operatorname{co} U=\operatorname{cl}(\operatorname{co} U)$.

We consider the robust vector optimization problem of the model [9], [10]:

(RVP) WMin $\left\{F(x): x \in C, G_{u}(x) \in-S, \forall u \in \mathcal{U}\right\}$,

where $K$ is a closed and convex cone in $Y$ with nonempty interior, and $S$ is a closed, convex cone in $Z, \mathcal{U}$ is an uncertainty set, $F: X \rightarrow Y^{\bullet}, G_{u}: X \rightarrow Z^{\bullet}$ are proper mappings, and $\emptyset \neq C \subset X$. The feasible set of (RVP) is

$$
A:=C \cap\left(\bigcap_{u \in \mathcal{U}} G_{u}^{-1}(-S)\right) .
$$

We assume through out this paper that $A \cap \operatorname{dom} F \neq \emptyset$.

Robust optimization provides a way to approach optimization problems with uncertain data. The subject has attracted attention of many mathematicians around the world in the last decades (see [2], 3], 4] and the comprehensive survey papers [5] and [15]). Many works devoted to the duality results on (both scalar and vector) robust optimization problems appeared in the literature (see [6], [7], [12], [13], [14], [16], [17], [20], and the references therein). In the recent years, the extension of Farkas-type results to systems with vector-valued functions, enables the authors to study the duality for vector optimization problems [8],[11], and also, the duality for robust vector optimization problems [9], [10].

Motivated by the works [9], 10, the present paper continues the study on the duality for robust vector problem of the models (RVP). The key results of the paper are the representations of the epigraph of the conjugate mapping $\left(F+I_{A}\right)^{*}$ via closure of the sectionally convex hull of a union of epigraphs of conjugate mappings of mappings from a family involving the data of the problem (RVP). With this better understanding on the presentation of epi $\left(F+I_{A}\right)^{*}$, we propose several new concrete qualification conditions, establish stable robust vector/convex vector Farkas lemmas. These results are then used used to establish new results on robust strong stable duality results for (RVP).

The paper is organized as follows: In Section 2, we recall some notations on weak supremum and weak infimum of a set in locally topological vector spaces ordered by closed convex cones with non-empty interiors, and some important properties of these notions. In Section 3 we introduce some basic mathematical tools which will be the key tools for the our study. We first introduce the notions of "uniformly $S^{+}$-concave of a family mappings and " $S^{+}$-uniform usc of a mapping. Notions of $k$-sectional convexity, $k$-sectional closedness, " $k$-sectional convex hull" of sets in a product space are then introduced together with some important properties of these notions. Section 4 is used the establishing various representations of the epigraph of the conjugate mapping $\left(F+I_{A}\right)^{*}$, epi $\left(F+I_{A}\right)^{*}$ via closure of sectional convex hull of a union of epigraphs of 
conjugate mappings involving in the problem (RVP). These results then play crucial roles in proving the main results sections that follows. Stable robust vector Farkas lemma are established in Section 5. Section 6 is left for duality results for robust vector problems (RVP). It is shown in Section 7 that when specifying the results obtained in Section 6 to some concrete classes of scalar robust problems (i.e., when $Y=\mathbb{R}$ ), our results still cover and extend several corresponding known ones in the literature.

\section{Preliminaries and notations}

We now recall some notions and properties that will be useful in the sequent.

Let $K \varsubsetneqq Y$ be a closed and convex cone in $Y$ with nonempty interion, i.e., int $K \neq \emptyset$. It is worth observing that $K+\operatorname{int} K=\operatorname{int} K$, and consequently,

$$
\left.\begin{array}{c}
y \in K \\
y^{\prime} \notin-\operatorname{int} K
\end{array}\right\} \Longrightarrow y+y^{\prime} \notin-\operatorname{int} K
$$

The next properties are useful in the sequel.

Lemma 2.1. Let $\left.y \in Y, k_{0} \in K, \lambda \in\right] 0,1[$ and $\alpha, \beta \in \mathbb{R}$. Then

$$
\left.\begin{array}{l}
y+\alpha k_{0} \notin-\operatorname{int} K \\
y+\beta k_{0} \notin-\operatorname{int} K
\end{array}\right\} \Longrightarrow y+[\lambda \alpha+(1-\lambda) \beta] k_{0} \notin-\operatorname{int} K .
$$

Proof. Let us assume that the statement in the right-hand side of (2.2) is false, i.e.,

$$
y+[\lambda \alpha+(1-\lambda) \beta] k_{0} \in-\operatorname{int} K .
$$

If $\alpha \leq \beta$ then $\alpha-[\lambda \alpha+(1-\lambda) \beta]=(1-\lambda)(\alpha-\beta) \leq 0$, which, together with the fact that $k_{0} \in K$, leads to

$$
\{\alpha-[\lambda \alpha+(1-\lambda) \beta]\} k_{0}=\alpha k_{0}-[\lambda \alpha+(1-\lambda) \beta] k_{0} \in-K .
$$

From (2.3) and (2.4), we get $y+\alpha k_{0} \in-\operatorname{int} K-K=-\operatorname{int} K$.

In case $\beta \leq \alpha$, one has $\beta-[\lambda \alpha+(1-\lambda) \beta]=\lambda(\beta-\alpha) \leq 0$. The same argument as above leads to $y+\beta k_{0} \in-$ int $K$. Consequently, (2.2) holds.

Lemma 2.2. Let $y \in Y$ and $k_{0} \in \operatorname{int} K$. Then, there exist $\bar{\alpha} \in \mathbb{R}$ such that for $\alpha \in \mathbb{R}$, it holds

$$
\alpha<\bar{\alpha} \Longleftrightarrow\left(y+\alpha k_{0} \in-\operatorname{int} K\right) .
$$

Proof. Let denote $P:=\left\{\alpha \in \mathbb{R}: y+\alpha k_{0} \in-\operatorname{int} K\right\}$.

- Observe firstly that $P \neq \emptyset$ and $P \neq \mathbb{R}$. Indeed, as $k_{0} \in \operatorname{int} K$, there exist balanced, convex neighborhoods $V_{1}$ and $V_{2}$ of $0_{Y}$ such that $k_{0}+V_{1} \in \operatorname{int} K$ and $-k_{0}+V_{2} \subset-\operatorname{int} K$, respectively. Taking $\lambda_{2}>0$ with $\lambda_{2} y \in V_{2}$, one has $-k_{0}+\lambda_{2} y \in-\operatorname{int} K$ or equivalently, $y-\frac{1}{\lambda_{2}} k_{0} \in-\operatorname{int} K$, that means $-\frac{1}{\lambda_{2}} \in P$, showing that $P \neq \emptyset$. Now, taking $\lambda_{1}>0$ such that $\lambda_{1} y \in V_{1}$. We then have $y+\lambda_{1} k_{0} \in \operatorname{int} K$ or equivalently, $y+\frac{1}{\lambda_{1}} k_{0} \in \operatorname{int} K$. As $K$ is a proper cone, $(-\operatorname{int} K) \cap(\operatorname{int} K)=\emptyset$, and hence, $y+\frac{1}{\lambda_{1}} k_{0} \notin-\operatorname{int} K$, or equivalently, $\frac{1}{\lambda_{1}} \notin P$ yielding $P \neq \mathbb{R}$. It is also worth mentioning that for any $\lambda>0$

\footnotetext{
${ }^{1}$ It is also called a proper cone.
} 
such that $\lambda<\lambda_{1}$ then $\lambda y \in V_{1}$ and, by the same argument as above we have $\frac{1}{\lambda} \notin P$, meaning that $P$ is bounded above.

So, one has $\bar{\alpha}:=\sup P \in \mathbb{R}$. We now prove that (2.5) holds.

- [£] Assume that $y+\alpha k_{0} \in-\operatorname{int} K$. Then, $\alpha \in P$ and hence, $\alpha \leq \bar{\alpha}=\sup P$. Now, if $\alpha=\bar{\alpha}$ then, as $y+\alpha k_{0} \in-$ int $K$, there is a neighborhood $V$ of $0_{Y}$ such that $y+\alpha k_{0}+V \subset-\operatorname{int} K$. Take $\epsilon>0$ such that $\epsilon k_{0} \in V$, one has $y+\alpha k_{0}+\epsilon k_{0}=$ $y+(\alpha+\epsilon) k_{0} \in-\operatorname{int} K$ which yields $\alpha+\epsilon \in P$. We then have $\alpha+\epsilon>\alpha=\bar{\alpha}=\sup P$, a contradiction. Consequently, $\alpha<\bar{\alpha}$ as expected.

- $[\Longrightarrow]$ Assume that $\alpha<\bar{\alpha}$. Then, as $\bar{\alpha}=\sup P$, there is $\alpha_{1} \in P$ such that $\alpha<\alpha_{1}$. As $\alpha_{1} \in P$, it holds $y+\alpha_{1} k_{0} \in-\operatorname{int} K$, and hence,

$$
y+\alpha k_{0}=\left(y+\alpha_{1} k_{0}\right)+\left(\alpha-\alpha_{1}\right) k_{0} \in-\operatorname{int} K-\operatorname{int} K=-\operatorname{int} K .
$$

The proof is complete.

In this paper, we shall use the two orderings generated by the cone $K$ : the weak ordering and the usual ordering, defined respectively by, for any $y_{1}, y_{2} \in Y$,

- weak ordering: $y_{1}<_{K} y_{2}$ if and only if $y_{1}-y_{2} \in-\operatorname{int} K$,

- usual ordering: $y_{1} \leqq_{K} y_{2}$ if and only if $y_{1}-y_{2} \in-K$.

We enlarge $Y$ by attaching a greatest element $+\infty_{Y}$ and a smallest element $-\infty_{Y}$ which do not belong to $Y$, and we denote $Y^{\bullet}:=Y \cup\left\{-\infty_{Y},+\infty_{Y}\right\}$. By convention, $-\infty_{Y}<_{K} y<_{K}+\infty_{Y}$ for any $y \in Y$. We also assume by convention that

$$
\begin{gathered}
-\left(+\infty_{Y}\right)=-\infty_{Y}, \quad-\left(-\infty_{Y}\right)=+\infty_{Y}, \\
\left(+\infty_{Y}\right)+y=y+\left(+\infty_{Y}\right)=+\infty_{Y}, \quad \forall y \in Y \cup\left\{+\infty_{Y}\right\} \\
\left(-\infty_{Y}\right)+y=y+\left(-\infty_{Y}\right)=-\infty_{Y}, \quad \forall y \in Y \cup\left\{-\infty_{Y}\right\}
\end{gathered}
$$

The sums $\left(-\infty_{Y}\right)+\left(+\infty_{Y}\right)$ and $\left(+\infty_{Y}\right)+\left(-\infty_{Y}\right)$ are not considered in this paper.

Given $\emptyset \neq M \subset Y^{\bullet}$, the following notions quoted from [7, Definition 7.4.1]) will be used throughout this paper.

- An element $\bar{v} \in Y^{\bullet}$ is said to be a weakly infimal element of $M$ if for all $v \in M$ we have $v \nless_{K} \bar{v}$ and if for any $\tilde{v} \in Y^{\bullet}$ such that $\bar{v}<_{K} \tilde{v}$, then there exists some $v \in M$ satisfying $v<_{K} \tilde{v}$. The set of all weakly infimal elements of $M$ is denoted by WInf $M$ and is called the weak infimum of $M$.

- An element $\bar{v} \in Y^{\bullet}$ is said to be a weakly supremal element of $M$ if for all $v \in M$ we have $\bar{v} \nless_{K} v$ and if for any $\tilde{v} \in Y^{\bullet}$ such that $\tilde{v}<_{K} \bar{v}$, then there exists some $v \in M$ satisfying $\tilde{v}<_{K} v$. The set of all weakly supremal elements of $M$ is denoted by WSup $M$ and is called the weak supremum of $M$.

- The weak minimum of $M$ is the set WMin $M=M \cap$ WInf $M$ and its elements are the weakly minimal elements of $M$. The weak maximum of $M$, WMax $M$, is defined similarly, WMax $M:=M \cap \mathrm{WSup} M$.

Weak infimum and weak supremum of the empty set is defined by convention as $\operatorname{WSup} \emptyset=\left\{-\infty_{Y}\right\}$ and $\operatorname{WInf} \emptyset=\left\{+\infty_{Y}\right\}$, respectively. 
It follows from the definition of $\operatorname{WSup} M$ that $\operatorname{WSup}(M+a)=a+\mathrm{WSup} M$ for all $M \subset Y^{\bullet}$ and $a \in Y$, and

$$
\begin{aligned}
+\infty_{Y} \in \operatorname{WSup} M & \Longleftrightarrow \operatorname{WSup} M=\left\{+\infty_{Y}\right\} \\
& \Longleftrightarrow \forall \tilde{v} \in Y, \exists v \in M: \tilde{v}<_{K} v .
\end{aligned}
$$

Next properties of sets of weak suprema and weak minima were quoted from [8, Proposition 2.1], [7, Proposition 7.4.3], and [20, Proposition 2.4].

Lemma 2.3. Assume that $\emptyset \neq M \subset Y$. Then it holds:

(a) WSup $M=\operatorname{cl}(M-\operatorname{int} K) \backslash(M-\operatorname{int} K)$,

(b) If WSup $M \neq\left\{+\infty_{Y}\right\}$ then WSup $M-\operatorname{int} K=M-\operatorname{int} K$,

(c) If WSup $M \neq\left\{+\infty_{Y}\right\}$ then the following decomposition of $Y$ holds:

$$
Y=(M-\operatorname{int} K) \cup(\text { WSup } M) \cup(\text { WSup } M+\operatorname{int} K),
$$

(d) If $\emptyset \neq M, N \subset Y^{\bullet}$, then

$$
\mathrm{WSup}(\mathrm{WSup} M+\mathrm{WSup} N)=\mathrm{WSup}(M+\mathrm{WSup} N)=\mathrm{WSup}(M+N) \text {. }
$$

It is worth noting that $\operatorname{WInf} M=-\operatorname{WSup}(-M)$ for all $M \subset Y^{\bullet}$. So, all the assertions in Lemma 2.3 still hold when all the terms WSup, WMax, int $K$, and $+\infty_{Y}$ are replaced by WInf, WMin, - int $K$, and $-\infty_{Y}$, respectively.

We denote by $\mathcal{L}(X, Y)$ the space of linear continuous mappings from $X$ to $Y$, and by $0_{\mathcal{L}}$ the zero element of $\mathcal{L}(X, Y)$ (i.e., $0_{\mathcal{L}}(x)=0_{Y}$ for all $\left.x \in X\right)$. The topology considered in $\mathcal{L}(X, Y)$ is the one defined by the point-wise convergence, i.e., $\left(L_{\alpha}\right)_{\alpha \in D} \subset$ $\mathcal{L}(X, Y)$ and $L \in \mathcal{L}(X, Y), L_{\alpha} \rightarrow L$ means that $L_{\alpha}(x) \rightarrow L(x)$ in $Y$ for all $x \in X$.

Given a vector-valued mapping $F: X \rightarrow Y^{\bullet}$, the effective domain of $F$ is defined by $\operatorname{dom} F:=\left\{x \in X: F(x) \neq+\infty_{Y}\right\}$, and the $K$-epigraph of $F$ is defined by $\operatorname{epi}_{K} F=\{(x, y) \in X \times Y: y \in F(x)+K\}$. As $K$ is fixed for the whole paper, we will write epi $F$ instead of epi $_{K} F$. We say that

- $F$ is proper if $\operatorname{dom} F \neq \emptyset$ and $-\infty_{Y} \notin F(X)$,

- $F$ is $K$-convex if epi $F$ is a convex subset of $X \times Y$,

- $F$ is $K$-epi closed if epi $F$ is a closed subset of $X \times Y$,

- $F$ is positively $K$-lower semi-continuous or $K$-lsc ( $K$-upper semi-continuous, resp.) if $y^{*} \circ F$ is lsc $\left(y^{*} \circ F\right.$ is usc, resp.) for all $y^{*} \in K^{+} \backslash\left\{0_{Y^{*}}\right\}$.

The conjugate mapping of $F$ is the set-valued map $F^{*}: \mathcal{L}(X, Y) \rightrightarrows Y^{\bullet}$ defined by $[$, Definition 2.8]

$$
F^{*}(L):=\operatorname{WSup}\{L(x)-F(x): x \in X\} .
$$

The domain of $F^{*}$ is $\operatorname{dom} F^{*}:=\left\{L \in \mathcal{L}(X, Y): F^{*}(L) \neq\left\{+\infty_{Y}\right\}\right\}$, the K-epigraph of $F^{*}$ is

$$
\text { epi } F^{*}:=\left\{(L, y) \in \mathcal{L}(X, Y) \times Y: y \in F^{*}(L)+K\right\} .
$$

It is useful to mention that if $F: X \rightarrow Y^{\bullet}$ is a proper mapping then epi $F^{*}$ is a closed subset of $\mathcal{L}(X, Y) \times Y$ [11, Lemma 3.5]. Moreover, it is established in [8, Theorem 3.1] that

$$
(L, y) \in \operatorname{epi} F^{*} \Longleftrightarrow(y-L(x)+F(x) \notin-\operatorname{int} K, \forall x \in \operatorname{dom} F) .
$$


The indicator mapping $I_{D}: X \rightarrow Y^{\bullet}$ of a set $D \subset X$ is defined by

$$
I_{D}(x)= \begin{cases}0_{Y}, & \text { if } x \in D \\ +\infty_{Y}, & \text { otherwise. }\end{cases}
$$

In the case where $Y=\mathbb{R}, I_{D}$ collapses to the usual indicator function $i_{D}$ of the set $D$ and the conjugate mapping $F^{*}$ collapses to the Fenchel conjugate function $F^{*}: X^{*} \rightarrow$ $\mathbb{R} \cup\{ \pm \infty\}$ with $F^{*}\left(x^{*}\right)=\sup _{x \in X}\left[\left\langle x^{*}, x\right\rangle-F(x)\right]$ for all $x^{*} \in X^{*}$. As usual, by $\Gamma(X)$ we denote the set of all proper, convex and lsc functions on $X$.

Let $S \neq \emptyset$ be a convex cone in $Z$ and $\leqq_{S}$ be the usual ordering on $Z$ induced by the cone $S$, i.e., $z_{1} \leqq_{S} z_{2}$ if and only if $z_{2}-z_{1} \in S$. We also enlarge $Z$ by attaching a greatest element $+\infty_{Z}$ and a smallest element $-\infty_{Z}$ which do not belong to $Z$, and define $Z^{\bullet}:=Z \cup\left\{-\infty_{Z},+\infty_{Z}\right\}$. In $Z^{\bullet}$ we adopt the same conventions as in (2.6). Moreover, we recall the cone of positive operators (see [1], 8]) and the cone of weak positive operators [1] respectively, as follows:

$$
\begin{aligned}
& \mathcal{L}_{+}(S, K):=\{T \in \mathcal{L}(Z, Y): T(S) \subset K\} \text { and } \\
& \mathcal{L}_{+}^{w}(S, K):=\{T \in \mathcal{L}(Z, Y): T(S) \cap(-\operatorname{int} K)=\emptyset\} .
\end{aligned}
$$

Lastly, for $T \in \mathcal{L}(Z, Y)$ and $G: X \rightarrow Z^{\bullet}$, the composite function $T \circ G: X \rightarrow Y^{\bullet}$ is defined as follows:

$$
(T \circ G)(x)= \begin{cases}T(G(x)), & \text { if } G(x) \in Z \\ +\infty_{Y}, & \text { if } G(x)=+\infty_{Z}\end{cases}
$$

\section{Basic tools: Sectional convexity and sectional closedness}

In this section, we will introduce notions of generalized convexity and closedness, namely, the so-called "sectional convexity" and "sectional closedness", respectively, and establish some basic properties of these notions. We introduce the notions of $\mathrm{S}^{+}$ uniformly usc of a mapping and uniformly $S^{+}$-concave of a family of mappings with their basic properties.

\subsection{Uniform $S^{+}$-concavity and $S^{+}$-uniformly upper semi-continuity}

Let $Z$ be a lcHtvs with a pre-order defined by a non-empty, closed and convex cone $S \subset Z$, and $\mathcal{U}$ be a topological space.

Definition 3.1. Let $\mathcal{G}, \mathcal{G}_{\nu}: \mathcal{U} \rightarrow Z \cup\left\{+\infty_{Z}\right\}$ for all $\nu \in I$.

- We say that the collection $\left(\mathcal{G}_{\nu}\right)_{\nu \in I}$ is uniformly $S^{+}$-concave if

$$
\begin{gathered}
\forall z_{1}^{*}, z_{2}^{*} \in S^{+}, \forall u_{1}, u_{2} \in \mathcal{U}, \exists z^{*} \in S^{+}, \exists u \in \mathcal{U} \text { such that } \\
\left(z_{1}^{*} \circ \mathcal{G}_{\nu}\right)\left(u_{1}\right)+\left(z_{2}^{*} \circ \mathcal{G}_{\nu}\right)\left(u_{2}\right) \leq\left(z^{*} \circ \mathcal{G}_{\nu}\right)(u), \forall \nu \in I .
\end{gathered}
$$

- We say that $\mathcal{G}$ is $S^{+}$-uniformly usc if, for any net $\left(z_{\alpha}^{*}, u_{\alpha}, r_{\alpha}\right)_{\alpha \in D} \subset S^{+} \times \mathcal{U} \times \mathbb{R}$ and $\left(z^{*}, u, r\right) \in S^{+} \times \mathcal{U} \times \mathbb{R}$,

$$
\left\{\begin{array}{l}
\left(z_{\alpha}^{*} \circ \mathcal{G}\right)\left(u_{\alpha}\right) \geq r_{\alpha}, \forall \alpha \in D \\
z_{\alpha}^{*} \stackrel{*}{\rightarrow} z^{*}, u_{\alpha} \rightarrow u, r_{\alpha} \rightarrow r
\end{array} \quad \Longrightarrow\left(z^{*} \circ \mathcal{G}\right)(u) \geq r .\right.
$$


The next example illustrates the meaning of the concept of "uniformly $S^{+}$-concave" and it is used in the proof of Corollary 7.4 in Section 7.

Example 3.1. Consider the case when $Z=\mathbb{R}$ and $S=\mathbb{R}_{+}$, and then, $Z^{*}=\mathbb{R}$ and $S^{+}=\mathbb{R}_{+}$. Assume that $\mathcal{U}$ is a convex subset of some topological vector space and let $g_{\nu}: \mathcal{U} \rightarrow \mathbb{R} \cup\{+\infty\}$ be a concave function for each $\nu \in I$. Then $\left(g_{\nu}\right)_{\nu \in I}$ is uniformly $\mathbb{R}_{+}$-concave. In deed, take $\lambda_{1}, \lambda_{2} \geq 0$ and $u_{1}, u_{2} \in \mathcal{U}$, we find $\lambda \geq 0$ and $u \in \mathcal{U}$ such that

$$
\lambda_{1} g_{\nu}\left(u_{1}\right)+\lambda_{2} g_{\nu}\left(u_{2}\right) \leq \lambda g_{\nu}(u), \quad \nu \in I .
$$

If $\lambda_{1}=\lambda_{2}=0$, we just take $\lambda=0$ and $u=u_{1}$. Assume that $\lambda_{1}>0$ or $\lambda_{2}>0$, or equivalently, $\lambda_{0}:=\lambda_{1}+\lambda_{2}>0$. For all $\nu \in I$, as $g_{\nu}$ is concave, one has

$$
\lambda_{1} g_{\nu}\left(u_{1}\right)+\lambda_{2} g_{\nu}\left(u_{2}\right)=\lambda_{0}\left(\frac{\lambda_{1}}{\lambda_{0}} g_{\nu}\left(u_{1}\right)+\frac{\lambda_{2}}{\lambda_{0}} g_{\nu}\left(u_{2}\right)\right) \leq \lambda_{0} g_{\nu}\left(\frac{\lambda_{1}}{\lambda_{0}} u_{1}+\frac{\lambda_{2}}{\lambda_{0}} u_{2}\right)
$$

(note that $\frac{\lambda_{1}}{\lambda_{0}}+\frac{\lambda_{2}}{\lambda_{0}}=1$ ). So, (3.2) follows by taking $\lambda=\lambda_{0}$ and $u=\frac{\lambda_{1}}{\lambda_{0}} u_{1}+\frac{\lambda_{2}}{\lambda_{0}} u_{2}$.

Lemma 3.1. (i) If $\mathcal{G}$ is $S^{+}$-uniformly usc then $\mathcal{G}$ is positively $S$-usc.

(ii) For $Z=\mathbb{R}$ and $S=\mathbb{R}_{+}, \mathcal{G}$ is $\mathbb{R}_{+}$-uniformly usc if and only if it is usc.

(iii) Assume that $\mathcal{U}_{i}$ is a topological space and that $\mathcal{G}_{i}: \mathcal{U}_{i} \rightarrow \mathbb{R} \cup\{+\infty\}$ is a usc function, for all $i=1, \ldots m$. Then, the mapping $\mathcal{G}: \mathcal{U}:=\prod_{i=1}^{m} \mathcal{U}_{i} \rightarrow \mathbb{R}^{m} \cup\left\{+\infty_{\mathbb{R}^{m}}\right\}$ defined by $\mathcal{G}\left(\left(u_{i}\right)_{i=1}^{m}\right)=\left(\mathcal{G}_{i}\left(u_{i}\right)\right)_{i=1}^{m}$ is $\mathbb{R}_{+}^{m}$-uniformly usc.

Proof. (i) Assume that $\mathcal{G}$ is $S^{+}$-uniformly usc. Take $z^{*} \in S^{+} \backslash\left\{0_{Z^{*}}\right\}$. As $\mathcal{G}$ is $S^{+}$-uniformly usc then, it holds, for all net $\left(u_{\alpha}, r_{\alpha}\right)_{\alpha \in D} \subset \mathcal{U} \times \mathbb{R}$ and $(u, r) \in \mathcal{U} \times \mathbb{R}$,

$$
\left\{\begin{array}{l}
\left(z^{*} \circ \mathcal{G}\right)\left(u_{\alpha}\right) \geq r_{\alpha}, \forall \alpha \in D \\
u_{\alpha} \rightarrow u, r_{\alpha} \rightarrow r
\end{array} \quad \Longrightarrow\left(z^{*} \circ \mathcal{G}\right)(u) \geq r\right.
$$

(let $z_{\alpha}^{*}=z^{*}$ for all $\left.\alpha \in D\right)$. This yields that the set $\left\{(u, r) \in \mathcal{U} \times \mathbb{R}:\left(z^{*} \circ \mathcal{G}\right)(u) \geq r\right\}$ is closed which also means that $z^{*} \circ \mathcal{G}$ is usc. So, $\mathcal{G}$ is positively $S$-usc.

(ii) If $\mathcal{G}$ is $\mathbb{R}_{+}$-uniformly usc then, according to $(i), \mathcal{G}$ is positively $\mathbb{R}_{+}$-usc yielding that $\mathcal{G}$ is usc. Conversely, assume that $\mathcal{G}$ is usc, we will prove that $\mathcal{G}$ is $\mathbb{R}_{+}$-uniformly usc. For this, take $\left(\lambda_{\alpha}, u_{\alpha}, r_{\alpha}\right)_{\alpha \in D} \subset \mathbb{R}_{+} \times \mathcal{U} \times \mathbb{R}$ and $(\lambda, u, r) \in \mathbb{R}_{+} \times \mathcal{U} \times \mathbb{R}$ satisfying

$$
\begin{gathered}
\lambda_{\alpha} \mathcal{G}\left(u_{\alpha}\right) \geq r_{\alpha}, \forall \alpha \in D \\
\text { and } \lambda_{\alpha} \rightarrow \lambda, u_{\alpha} \rightarrow u, r_{\alpha} \rightarrow r,
\end{gathered}
$$

we need to show that $\lambda \mathcal{G}(u) \geq r$.

If $\lambda>0$ then, for all $\alpha$ large enough, $\lambda_{\alpha}>0$ and hence (3.3) yields $\mathcal{G}\left(u_{\alpha}\right) \geq \frac{r_{\alpha}}{\lambda_{\alpha}}$. Passing to the limit one gets $\mathcal{G}(u) \geq \frac{r}{\lambda}$ (as $\mathcal{G}$ is usc), or equivalently, $\lambda \mathcal{G}(u) \geq r$.

If $\lambda=0$ then as $\mathcal{G}$ is usc and $u_{\alpha} \rightarrow u$, one has $\mathcal{G}\left(u_{\alpha}\right)<\mathcal{G}(u)+1$ for $\alpha$ large enough. So, from (3.3) , one has, for all $\alpha$ large enough, $\lambda_{\alpha}(\mathcal{G}(u)+1) \geq r_{\alpha}$. This leads to $r \leq 0$ (as $\lambda_{\alpha} \rightarrow 0$ and $r_{\alpha} \rightarrow r$ ), and hence, one has $\lambda \mathcal{G}(u)=0 \geq r$.

(iii) Take the net $\left(z_{\alpha}^{*}, u_{\alpha}, r_{\alpha}\right)_{\alpha \in D} \subset \mathbb{R}_{+}^{m} \times \mathcal{U} \times \mathbb{R}$ and $\left(z^{*}, u, r\right) \in \mathbb{R}_{+}^{m} \times \mathcal{U} \times \mathbb{R}$ with 
$z_{\alpha}^{*}=\left(\lambda_{i}^{\alpha}\right)_{i=1}^{m}, u_{\alpha}=\left(u_{i}^{\alpha}\right)_{i=1}^{m}, z^{*}=\left(\lambda_{i}\right)_{i=1}^{m}$ and $u=\left(u_{i}\right)_{i=1}^{m}$, and assume that

$$
\begin{gathered}
\sum_{i=1}^{m} \lambda_{i}^{\alpha} \mathcal{G}_{i}\left(u_{i}^{\alpha}\right) \geq r_{\alpha}, \forall \alpha \in D \\
\lambda_{i}^{\alpha} \rightarrow \lambda_{i}, u_{i}^{\alpha} \rightarrow u_{i}, \forall i=1, \ldots, m ; \quad r_{\alpha} \rightarrow r .
\end{gathered}
$$

We will prove that $\sum_{i=1}^{m} \lambda_{i} \mathcal{G}_{i}\left(u_{i}\right) \geq r$.

As $r_{\alpha} \rightarrow r$, there is $M>0$ such that $\left|r_{\alpha}\right| \leq M$ for all $\alpha \in D$. For all $i=1, \ldots, m$, as $\lambda_{i}^{\alpha} \rightarrow \lambda_{i}, u_{i}^{\alpha} \rightarrow u_{i}, \mathcal{G}_{i}$ is usc, there exists $M_{i}$ such that $\lambda_{i}^{\alpha} \mathcal{G}_{i}\left(u_{i}^{\alpha}\right) \leq M_{i}$ for all $\alpha \in D$.

Now, take $i \in\{1, \ldots, m-1\}$. It follows from (3.5) that

$$
M_{i} \geq \lambda_{i}^{\alpha} \mathcal{G}_{i}\left(u_{i}^{\alpha}\right) \geq r_{\alpha}-\sum_{j \in\{1, \ldots . m\} \backslash\{i\}} \lambda_{j}^{\alpha} \mathcal{G}_{j}\left(u_{j}^{\alpha}\right) \geq-M-\sum_{j \in\{1, \ldots m\} \backslash\{i\}} M_{j}, \quad \forall \alpha \in D
$$

So, we can suppose that $\gamma_{i}^{\alpha}:=\lambda_{i}^{\alpha} \mathcal{G}_{i}\left(u_{i}^{\alpha}\right) \rightarrow \gamma_{i}$. One then has

$$
\lambda_{i}^{\alpha} \mathcal{G}_{i}\left(u_{i}^{\alpha}\right) \geq \gamma_{i}^{\alpha}, \forall \alpha \in D \text { and } \lambda_{i}^{\alpha} \rightarrow \lambda_{i}, u_{i}^{\alpha} \rightarrow u_{i}, \gamma_{i}^{\alpha} \rightarrow \gamma_{i} .
$$

which, together with the fact that $\mathcal{G}_{i}$ is usc, yields $\lambda_{i} \mathcal{G}_{i}\left(u_{i}\right) \geq \gamma_{i}$ (see the proof of $(i i)$ ).

As $r_{\alpha} \rightarrow r$ and $\gamma_{i}^{\alpha} \rightarrow \gamma_{i}$ for all $i=1, \ldots m-1$, we have $r_{\alpha}-\sum_{i=1}^{m-1} \gamma_{i}^{\alpha} \rightarrow r-\sum_{i=1}^{m-1} \gamma_{i}$. Moreover, according to (3.5),

$$
\lambda_{m}^{\alpha} \mathcal{G}_{m}\left(u_{m}^{\alpha}\right) \geq r_{\alpha}-\sum_{i=1}^{m-1} \gamma_{i}^{\alpha}, \forall \alpha \in D .
$$

Consequently, as $\lambda_{m}^{\alpha} \rightarrow \lambda_{m}, u_{m}^{\alpha} \rightarrow u_{m}$, and as $\mathcal{G}_{m}$ is usc, it holds $\lambda_{m} \mathcal{G}_{m}\left(u_{m}\right) \geq$ $r-\sum_{i=1}^{m-1} \gamma_{i}$ (see again the proof of $(i i)$ ). So, $\sum_{i=1}^{m} \lambda_{i} \mathcal{G}_{i}\left(u_{i}\right) \geq \sum_{i=1}^{m-1} \gamma_{i}+r-\sum_{i=1}^{m-1} \gamma_{i}=r$ and we are done.

\subsection{Sectional convexity and sectional closedness in topological vector spaces}

Let $E$ be a topological vector space with $E_{0}$ being its closed subspace.

Definition 3.2 (Sectional convexity). We say that the subset $N \subset E$ is $E_{0}$-sectionally convex if $N \cap\left(E_{0}+v\right)$ is convex for all $v \in E$.

It is worth noting that if $N$ is a convex set then $N \cap\left(E_{0}+v\right)$ is convex for all $v \in E$, and hence, $N$ is also a $E_{0}$-sectionally convex set. The converse, however, in general is not true, for instance, if $E=\mathbb{R}^{2}$ and $E_{0}=\{0\} \times \mathbb{R}$, then the set $N=\left\{(\alpha, \beta) \in \mathbb{R}^{2}\right.$ : $\left.\alpha^{2} \leq \beta \leq \alpha^{2}+1\right\}$ is $E_{0}$-sectionally convex but it is obviously not convex.

It is easy to see that the intersection of all $E_{0}$-sectionally convex subsets of $E$ containing $\emptyset \neq N \subset E$ is an $E_{0}$-sectionally convex subset of $E$ that contains $N$, which is called the $E_{0}$-sectionally convex hull of $N$, and denoted by $\operatorname{sco}_{E_{0}} N$. Clearly, $\operatorname{sco}_{E_{0}} N$ is the smallest $E_{0}$-sectionally convex subset of $E$ that contains $N$. Moreover, it is easy to see that

- $\mathrm{sco}_{E_{0}} N \subset \operatorname{co} N$,

- $N$ is $E_{0}$-sectionally convex if and only if $\operatorname{sco}_{E_{0}} N=N$,

- If $N$ is $E_{0}$-sectionally convex then $\mathrm{cl} N$ is $E_{0}$-sectionally convex. 
Moreover, when $E_{0}=E$, the concepts of " $E$-sectionally convex" and " $E$-sectionally convex hull" go back to the usual ones "convex" and "convex hull" in convex analysis, respectively.

The next proposition gives a presentation of $E_{0}$-sectionally convex hull of a set via the convex hull.

Proposition 3.1. Let $\emptyset \neq N \subset E$. Then

$$
\operatorname{sco}_{E_{0}} N=\bigcup_{v \in E} \operatorname{co}\left(N \cap\left(E_{0}+v\right)\right) .
$$

Proof. Denote the set in right-hand side of (3.7) by $M$. To prove (3.7), it is sufficient to check that (i) $N \subset M$, (ii) $M$ is a $E_{0}$-sectionally convex set, and (iii) $M \subset M^{\prime}$ for all $E_{0}$-sectionally convex subset $M^{\prime}$ of $E$ that contains $N$.

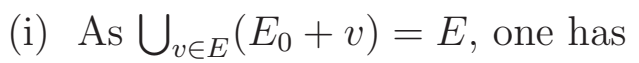

$$
N=N \cap\left(\bigcup_{v \in E}\left(E_{0}+v\right)\right)=\bigcup_{v \in E}\left[N \cap\left(E_{0}+v\right)\right] \subset \bigcup_{v \in E} \operatorname{co}\left[N \cap\left(E_{0}+v\right)\right]=M
$$

(ii) We now prove that $M$ is $E_{0}$-sectionally convex. For this, take any $\bar{v} \in E$, we will show that $M \cap\left(E_{0}+\bar{v}\right)$ is a convex set. Let us represent $M=M_{1} \cup M_{2}$ with

$$
M_{1}:=\bigcup_{v \in E_{0}+\bar{v}} \operatorname{co}\left(N \cap\left(E_{0}+v\right)\right), \quad M_{2}:=\bigcup_{v \in E \backslash\left(E_{0}+\bar{v}\right)} \operatorname{co}\left(N \cap\left(E_{0}+v\right)\right) .
$$

As $E_{0}$ is a subspace of $E$, it is easy to check that $E_{0}+v=E_{0}+\bar{v}$ whenever $v \in E_{0}+\bar{v}$ and $\left(E_{0}+v\right) \cap\left(E_{0}+\bar{v}\right)=\emptyset$ whenever $v \notin E_{0}+\bar{v}$. This entails $M_{1}=\operatorname{co}\left(N \cap\left(E_{0}+\bar{v}\right)\right)$ and

$$
\begin{aligned}
M_{2} \cap\left(E_{0}+\bar{v}\right) & =\left(\bigcup_{v \in E \backslash\left(E_{0}+\bar{v}\right)} \operatorname{co}\left(N \cap\left(E_{0}+v\right)\right)\right) \cap\left(E_{0}+\bar{v}\right) \\
& \subset\left(\bigcup_{v \in E \backslash\left(E_{0}+\bar{v}\right)}\left(E_{0}+v\right)\right) \cap\left(E_{0}+\bar{v}\right)=\emptyset .
\end{aligned}
$$

Note that the last inclusion follows from the fact that

$$
\operatorname{co}\left(N \cap\left(E_{0}+v\right)\right) \subset E_{0}+v, \quad \forall v \in E
$$

as $E_{0}+v$ is a convex subset containing $N \cap\left(E_{0}+v\right)$. Consequently,

$$
M \cap\left(E_{0}+\bar{v}\right)=\left(\operatorname{co}\left(N \cap\left(E_{0}+\bar{v}\right)\right) \cap\left(E_{0}+\bar{v}\right)\right) \cup \emptyset=\operatorname{co}\left(N \cap\left(E_{0}+\bar{v}\right)\right)
$$

(see (3.8)). So, $M \cap\left(E_{0}+\bar{v}\right)$ is a convex set.

(iii) Now, assume that $M^{\prime}$ is an $E_{0}$-sectionally convex subset containing $N$, we will show that $M \subset M^{\prime}$. Take an arbitrary $w \in M$. Then, by the definition of $M$, there exists $v \in E$ such that $w \in \operatorname{co}\left(N \cap\left(E_{0}+v\right)\right)$. On the other hand, as $M^{\prime}$ is an $E_{0}$-sectionally convex set containing $N$, the set $M^{\prime} \cap\left(E_{0}+v\right)$ is convex and contains $N \cap\left(E_{0}+v\right)$. So, $M^{\prime} \cap\left(E_{0}+v\right) \supset \operatorname{co}\left(N \cap\left(E_{0}+v\right)\right)$ which yields $w \in M^{\prime}$. The proof is complete. 
Definition 3.3 (Sectional closedness). We say that the subset $N \subset E$ is $E_{0}$-sectionally closed if for all $v \in E$ the set $N \cap\left(E_{0}+v\right)$ is closed in $E$.

It is also easy to see that a closed subset of $E$ is always $E_{0}$-sectionally closed subset of $E$ for any closed subspace $E_{0}$ of $E$. In general, however, the converse is not true. For instance, consider $E=\mathbb{R}^{2}$ and $E_{0}=\{0\} \times \mathbb{R}$, then the set $(0,1) \times[0,1]$ is $E_{0}$-sectionally closed but it is not a closed subset of $\mathbb{R}^{2}$.

For $N \subset E$, the intersection of all $E_{0}$-sectionally closed (resp., $E_{0}$-sectionally closed and convex) subset of $E$ containing $N$ is called the $E_{0}$-sectional closure (resp., $E_{0^{-}}$ sectionally closed and convex hull of $N$ ) of $N$, and is denoted by $\operatorname{scl}_{E_{0}} N$ (resp., by $\left.\operatorname{sclco}_{E_{0}} N\right)$.

Proposition 3.2. Let $\emptyset \neq N \subset Y$.

(i) $\operatorname{scl}_{E_{0}} N=\bigcup_{v \in E} \operatorname{cl}\left(N \cap\left(E_{0}+v\right)\right)$,

(ii) If $N$ is $E_{0}$-sectionally convex then $\operatorname{scl}_{E_{0}} N$ is $E_{0}$-sectionally convex,

(iii) $\operatorname{sclco}_{E_{0}} N=\operatorname{scl}_{E_{0}}\left(\operatorname{sco}_{E_{0}} N\right)=\bigcup_{v \in E} \operatorname{clco}\left(N \cap\left(E_{0}+v\right)\right)$.

Proof. (i) The conclusion follows from the same argument as in the proof of Proposition 3.1

(ii) Assume that $N$ is a $E_{0}$-sectionally convex. Take $\bar{v} \in E$ and we will prove that $\left(\operatorname{scl}_{E_{0}} N\right) \cap\left(E_{0}+\bar{v}\right)$ is convex. It follows from the same argument as in the second part of the proof of Proposition 3.1 and from (i) that

$$
\operatorname{scl}_{E_{0}} N=\left[\bigcup_{v \in E_{0}+\bar{v}} \operatorname{cl}\left(N \cap\left(E_{0}+v\right)\right)\right] \bigcup\left[\bigcup_{v \in E \backslash\left(E_{0}+\bar{v}\right)} \operatorname{cl}\left(N \cap\left(E_{0}+v\right)\right)\right],
$$

and hence,

$$
\left(\operatorname{scl}_{E_{0}} N\right) \cap\left(E_{0}+\bar{v}\right)=\left[M_{1} \cap\left(E_{0}+\bar{v}\right)\right] \cup\left[M_{2} \cap\left(E_{0}+\bar{v}\right)\right],
$$

where $M_{1}:=\bigcup_{v \in E_{0}+\bar{v}} \operatorname{cl}\left(N \cap\left(E_{0}+v\right)\right)$ and $M_{2}:=\bigcup_{v \in E \backslash\left(E_{0}+\bar{v}\right)} \operatorname{cl}\left(N \cap\left(E_{0}+v\right)\right)$. Use the similar argument as in the proof of Proposition 3.1 we can show that $M_{1} \cap\left(E_{0}+\bar{v}\right)=$ $\operatorname{cl}\left(N \cap\left(E_{0}+\bar{v}\right)\right)$ and $M_{2} \cap\left(E_{0}+\bar{v}\right)=\emptyset$, and hence, $\left(\operatorname{scl}_{E_{0}} N\right) \cap\left(E_{0}+\bar{v}\right)=\operatorname{cl}\left(N \cap\left(E_{0}+\bar{v}\right)\right)$. On the other hand, as $N$ is $E_{0}$-sectionally convex, the set $N \cap\left(E_{0}+\bar{v}\right)$ is convex. So, $\operatorname{cl}\left(N \cap\left(E_{0}+\bar{v}\right)\right)$ is convex, as well, and we are done.

(iii) Observe that $\operatorname{scl}_{E_{0}}\left(\mathrm{sco}_{E_{0}} N\right)=\operatorname{sclco}_{E_{0}} N$. Indeed, as sclco $E_{0} N$ is a $E_{0}$-sectionally convex subset containing $N$, one has $\operatorname{sclco}_{E_{0}} N \supset \operatorname{sco}_{E_{0}} N$. Note that $\operatorname{sclco}_{E_{0}} N$ is also $E_{0}$-sectionally closed, so $\operatorname{sclco}_{E_{0}} N \supset \operatorname{scl}_{E_{0}}\left(\operatorname{sco}_{E_{0}} N\right)$.

Conversely, by Proposition $\left[3.2(i i), \mathrm{scl}_{E_{0}}\left(\mathrm{sco}_{E_{0}} N\right)\right.$ is a $E_{0^{-}}$-sectionally closed and $E_{0^{-}}$ sectionally convex subset containing $N$ yielding $\operatorname{scl}_{E_{0}}\left(\operatorname{sco}_{E_{0}} N\right) \supset \operatorname{sclco}_{E_{0}} N$.

Lastly, the equality $\operatorname{sclco}_{E_{0}} N=\bigcup_{v \in E} \operatorname{cl} \operatorname{co}\left(N \cap\left(E_{0}+v\right)\right)$ follows from the same argument as in the proof of Proposition 3.1 .

\subsection{Sectional convexity and sectional closedness of epigraphs of conjugate mappings}

Let $k \in Y \backslash\left\{0_{Y}\right\}$. For $x^{*} \in X^{*}$, we define the mapping $k \cdot x^{*}: X \rightarrow Y$ by

$$
\left(k \cdot x^{*}\right)(x)=\left\langle x^{*}, x\right\rangle k, \forall x \in X \text {. }
$$


Throughout this paper, we are dealing with the space $E=\mathcal{L}(X, Y) \times Y$ and its subspace

$$
E_{k}=k \cdot\left(X^{*} \times \mathbb{R}\right):=\left\{\left(k \cdot x^{*}, r k\right): x^{*} \in X^{*}, r \in \mathbb{R}\right\} .
$$

So, for the sake of simplicity, a subset $\mathcal{E} \subset \mathcal{L}(X, Y) \times Y$ is $E_{k}$-sectionally convex then we say that it is $k$-sectionally convex and for the $E_{k}$-sectionally convex hull of $\mathcal{E}$, we write $\mathrm{sco}_{k} \mathcal{E}$ (instead of $\mathrm{sco}_{E_{k}} \mathcal{E}$ ) and call it $k$-sectionally convex hull of $\mathcal{E}$. The same way applies to the " $E_{k}$-sectional closedness" of $\mathcal{E}$ as well.

Turning back to the case when $Y=\mathbb{R}$, for each $\alpha \in \mathbb{R} \backslash\{0\}$ (playing the role of $k)$, one has $\alpha \cdot\left(X^{*} \times \mathbb{R}\right)+\left(\bar{x}^{*}, \bar{r}\right)=X^{*} \times \mathbb{R}$ for all $\left(\bar{x}^{*}, \bar{r}\right) \in X^{*} \times \mathbb{R}$. So, for given $\mathcal{E} \subset X^{*} \times \mathbb{R}$, it holds $\mathcal{E} \cap\left(\alpha \cdot\left(X^{*} \times \mathbb{R}\right)+\left(\bar{x}^{*}, \bar{r}\right)\right)=\mathcal{E}$ for any $\left(\bar{x}^{*}, \bar{r}\right) \in X^{*} \times \mathbb{R}$. In other words, in this case, the notions of " $\alpha$-sectionally convex", " $\alpha$-sectionally closed", " $\alpha$-sectionally convex hull", and " $\alpha$-sectional closure" collapse to the usual "convex", "closed", "convex hull", and "closure" in convex analysis, respectively.

It is worth noticing that epi $F^{*}$, in general, is not a convex subset of $\mathcal{L}(X, Y) \times Y$ even when $F$ is a linear continuous mapping (see [11, Example 2.6]). However, as we will see in the next proposition, it is always $k$-sectionally convex for any $k \in K \backslash\left\{0_{Y}\right\}$.

Proposition 3.3. Let $F: X \rightarrow Y^{\bullet}$ be a proper mapping. Then, epi $F^{*}$ is $k$-sectionally convex for each $k \in K \backslash\left\{0_{Y}\right\}$.

Proof. Let $k \in K \backslash\left\{0_{Y}\right\},(L, y) \in \mathcal{L}(X, Y) \times Y$, and let $E_{k}=k \cdot\left(X^{*} \times \mathbb{R}\right)$ as in (3.10). We will prove that $\left(\right.$ epi $\left.F^{*}\right) \cap\left[E_{k}+(L, y)\right]$ is a convex subset of $\mathcal{L}(X, Y) \times Y$. For this, take $a_{1}, a_{2} \in\left(\right.$ epi $\left.\left.F^{*}\right) \cap\left[E_{k}+(L, y)\right], \lambda \in\right] 0,1\left[\right.$, it suffices to show that $\lambda a_{1}+(1-\lambda) a_{2} \in$ epi $F^{*}$. As $a_{i} \in E_{k}+(L, y)$, there exists $\left(x_{i}^{*}, r_{i}\right) \in X^{*} \times \mathbb{R}$ such that $a_{i}=k\left(x_{i}^{*}, r_{i}\right)+(L, y)$, $i=1,2$. On the other hand, as $a_{i} \in$ epi $F^{*}$, one has (see (2.9))

$$
y+r_{i} k-L(x)-\left\langle x_{i}^{*}, x\right\rangle k+F(x) \notin-\operatorname{int} K, \forall x \in \operatorname{dom} F .
$$

Then, according to Lemma 2.1, we get $y+\left[\lambda r_{1}+(1-\lambda) r_{2}\right] k-L(x)-\left\langle\lambda x_{1}^{*}+(1-\lambda) x_{2}^{*}, x\right\rangle k+F(x) \notin-\operatorname{int} K, \forall x \in \operatorname{dom} F$ which, again by (2.9) , yields $\lambda a_{1}+(1-\lambda) a_{2} \in$ epi $F^{*}$ and the proof is complete.

It is worth observing that $\left.k \cdot\left(X^{*} \times \mathbb{R}\right)=(-k) \dot{(} X^{*} \times \mathbb{R}\right)$ for any $k \in K$. So, by Proposition 3.3, epi $F^{*}$ is $k$-sectionally convex for all $k \in[K \cup(-K)] \backslash\left\{0_{Y}\right\}$. However, the conclusion might not be true when $k \notin K \cup(-K)$ as shown in the next example.

Example 3.2. Take $X=\mathbb{R}, Y=\mathbb{R}^{2}, K=\mathbb{R}_{+}^{2}, F: \mathbb{R} \rightarrow \mathbb{R}^{2}$ the null mapping. Then $\mathcal{L}(X, Y)=\mathbb{R}^{2}$ and we get from Example 2.2 in [11] that epi $F^{*}=\bigcup_{i=1}^{4} N_{i}$ with

$$
\begin{aligned}
& N_{1}=\left\{\left(0,0, y_{1}, y_{2}\right): y_{1} \geq 0 \text { or } y_{2} \geq 0\right\}, \quad N_{2}=\left\{\left(\alpha, \beta, y_{1}, y_{2}\right): \alpha \beta<0, y_{2} \geq \frac{\beta}{\alpha} y_{1}\right\}, \\
& N_{3}=\left\{\left(\alpha, 0, y_{1}, y_{2}\right): \alpha \neq 0, y_{2} \geq 0\right\}, \quad N_{4}=\left\{\left(0, \beta, y_{1}, y_{2}\right): \beta \neq 0, y_{1} \geq 0\right\} .
\end{aligned}
$$

Now, take $k=(1,-1), E_{k}=k \cdot\left(X^{*} \times \mathbb{R}\right), L=(0,0)$ and $y=(0,-1)$. Then $E_{k}+(L, y)=\left\{\left(\alpha,-\alpha, y_{1},-y_{1}-1\right): \alpha, y_{1} \in \mathbb{R}\right\}$, and hence,

$$
\left(\text { epi } F^{*}\right) \cap\left[E_{k}+(L, y)\right]=\left\{\left(0,0, y_{1},-y_{1}-1\right): y_{1} \in \mathbb{R}, y_{1} \geq 0 \text { or } y_{1} \leq-1\right\},
$$

showing that (epi $\left.F^{*}\right) \cap\left[E_{k}+(L, y)\right]$ is not a convex set, and consequently, epi $F^{*}$ is not $(1,-1)$-sectionally convex. 
Proposition 3.4. Let $F: X \rightarrow Y^{\bullet}, G: X \rightarrow Z^{\bullet}$ be proper mappings, and $k \in K \backslash$ $\left\{0_{Y}\right\}$. Then the set $\bigcup_{z \in S^{+}} \operatorname{epi}\left(F+\left(k \cdot z^{*}\right) \circ G\right)^{*}$ is $k$-sectionally convex, where $k \cdot z^{*} \in$ $\mathcal{L}(Z, Y)$ is the mapping defined as in (3.9).

Proof. Let $E_{k}=k \cdot\left(X^{*} \times \mathbb{R}\right)$ (defined by $(\underline{3.10})$ ), and set

$$
\mathcal{M}_{k}:=\bigcup_{z^{*} \in S^{+}} \operatorname{epi}\left(F+\left(k \cdot z^{*}\right) \circ G\right)^{*} .
$$

Take $(L, y) \in \mathcal{L}(X, Y) \times Y$ and we will show that the set $\mathcal{M}_{k} \cap\left[E_{k}+(L, y)\right]$ is convex. The proof goes parallelly as that of Proposition 3.3. Take $a_{1}, a_{2} \in \mathcal{M}_{k} \cap\left(E_{k}+(L, y)\right)$, $\lambda \in] 0,1\left[\right.$, it suffices to show that $\lambda a_{1}+(1-\lambda) a_{2} \in \mathcal{M}_{k}$. As $a_{i} \in \mathcal{M}_{k} \cap\left(E_{k}+(L, y)\right)$, there exist $\left(x_{i}^{*}, r_{i}\right) \in X^{*} \times \mathbb{R}$ and $z_{i}^{*} \in S^{+}$such that $a_{i}=k\left(x_{i}^{*}, r_{i}\right)+(L, y) \in \operatorname{epi}\left(F+\left(k \cdot z^{*}\right) \circ G\right)^{*}$ for $i=1,2$. By (2.9), one has

$$
y+r_{i} k-L(x)-\left\langle x_{i}^{*}, x\right\rangle k+F(x)+\left(z_{i}^{*} \circ G\right)(x) k \notin-\operatorname{int} K, \forall x \in X, \forall i=1,2 .
$$

It now follows from Lemma 2.1 that

$\lambda a_{1}+(1-\lambda) a_{2}=y+\bar{r} k-L(x)-\left\langle\bar{x}^{*}, x\right\rangle k+F(x)+\left(\bar{z}^{*} \circ G\right)(x) k \notin-\operatorname{int} K, \forall x \in X,(3.11)$ where $\bar{r}=\lambda r_{1}+(1-\lambda) r_{2}, \bar{x}^{*}=\lambda x_{1}^{*}+\left(1-\lambda_{2}\right) x_{2}^{*}$, and $\bar{z}^{*}:=\lambda z_{1}^{*}+(1-\lambda) z_{2}^{*}$ (note that $\bar{z}^{*} \in S^{+}$as $\left.z_{1}^{*}, z_{2}^{*} \in S^{+}\right)$. Again, by (2.9), (3.11) means that $\lambda a_{1}+(1-\lambda) a_{2} \in$ $\operatorname{epi}\left(F+\left(k \cdot \bar{z}^{*}\right) \circ G\right)^{*} \subset \mathcal{M}_{k}$ and the proof is complete.

Remark 3.1. According to [11, Lemma 3.6], for any proper mapping $F: X \rightarrow Y^{\bullet}$, the set epi $F^{*}$ is always closed and consequently, it is $k$-sectionally closed for each $k \in Y \backslash\{0\}$.

\section{Epigraphs of conjugate mappings via sectionally convex hulls}

We are now concerning the robust vector optimization problem of the model [9], [10]:

(RVP) WMin $\left\{F(x): x \in C, G_{u}(x) \in-S, \forall u \in \mathcal{U}\right\}$,

where, as in previous sections, $X, Y, Z$ are lcHtvs, $K$ is a closed and convex cone in $Y$ with nonempty interior, and $S$ is a closed, convex cone in $Z, \mathcal{U}$ is an uncertainty set, $F: X \rightarrow Y^{\bullet}, G_{u}: X \rightarrow Z^{\bullet}$ are proper mappings, and $\emptyset \neq C \subset X$. The feasible set of $(\mathrm{RVP})$ is

$$
A:=C \cap\left(\bigcap_{u \in \mathcal{U}} G_{u}^{-1}(-S)\right) .
$$

We assume through out this paper that $A \cap \operatorname{dom} F \neq \emptyset$.

In this section we will establish various representations of the epigraph of the conjugate mapping $\left(F+I_{A}\right)^{*}$, epi $\left(F+I_{A}\right)^{*}$. The representations hold under "closure" signs and without any constraint qualification conditions and so they are called asymptotic representations. These representations will play a crucial role in establishing the main results of the next sections: robust vector Farkas-type results and duality for the problem (RVP). 
Concerning the problem(RVP), we recall the qualifying set [9] and the weak qualifying set [10] defined respectively as follows:

$$
\begin{aligned}
& \mathcal{A}:=\bigcup_{(T, u) \in \mathcal{L}_{+}(S, K) \times \mathcal{U}} \operatorname{epi}\left(F+I_{C}+T \circ G_{u}\right)^{*}, \\
& \mathcal{B}:=\bigcup_{(T, u) \in \mathcal{L}_{+}^{w}(S, K) \times \mathcal{U}}\left(\bigcap_{v \in I_{-S}^{*}(T)}\left[\operatorname{epi}\left(F+I_{C}+T \circ G_{u}\right)^{*}+\left(0_{\mathcal{L}}, v\right)\right]\right) .
\end{aligned}
$$

For $k \in \operatorname{int} K$, we now introduce another qualifying set $\mathcal{A}_{k}$ defined by

$$
\mathcal{A}_{k}:=\bigcup_{\left(z^{*}, u\right) \in S^{+} \times \mathcal{U}} \operatorname{epi}\left(F+I_{C}+\left(k \cdot z^{*}\right) \circ G_{u}\right)^{*},
$$

where $k \cdot z^{*}: Z \rightarrow Y$ defined by $\left(k \cdot z^{*}\right)(z)=\left\langle z^{*}, z\right\rangle k$ for all $z \in Z$ (see also (3.9)).

In the case when $Y=\mathbb{R}$ and $K=\mathbb{R}_{+}$all the sets $\mathcal{A}, \mathcal{B}$, and $\mathcal{A}_{k}$ collapse to the usual qualifying set (see [12]) $\bigcup_{\left(z^{*}, u\right) \in S^{+} \times \mathcal{U}} \operatorname{epi}\left(F+i_{C}+z^{*} \circ G_{u}\right)^{*}$.

The relations between these sets and epi $\left(F+I_{A}\right)^{*}$ are given in the next proposition.

Proposition 4.1. It holds $\operatorname{epi}\left(F+I_{A}\right)^{*} \supset \mathcal{B} \supset \mathcal{A} \supset \mathcal{A}_{k}$ for all $k \in \operatorname{int} K$.

Proof. It is easy to see that $k \cdot z^{*} \in \mathcal{L}_{+}(S, K)$ whenever $z^{*} \in S^{+}$and $k \in \operatorname{int} K$. So,

$$
\mathcal{A}_{k} \subset \mathcal{A}, \forall k \in \operatorname{int} K \text {. }
$$

Now, for each $u \in \mathcal{U}$, let $A_{u}:=C \cap G_{u}^{-1}(-S)$. Repeat the same argument as in the first part of the proof of Theorem 4.2 (namely, the proof of (27)) in [11], we get for each $u \in \mathcal{U}$,

Consequently,

$$
\begin{aligned}
\operatorname{epi}\left(F+I_{A_{u}}\right)^{*} & \supset \bigcup_{T \in \mathcal{L}_{+}^{w}(S, K)}\left(\bigcap_{v \in I_{-S}^{*}(T)}\left[\operatorname{epi}\left(F+I_{C}+T \circ G_{u}\right)^{*}+\left(0_{\mathcal{L}}, v\right)\right]\right) \\
& \supset \bigcup_{T \in \mathcal{L}_{+}(S, K)} \operatorname{epi}\left(F+I_{C}+T \circ G_{u}\right)^{*} .
\end{aligned}
$$

$$
\bigcup_{u \in \mathcal{U}} \operatorname{epi}\left(F+I_{A_{u}}\right)^{*} \supset \mathcal{B} \supset \mathcal{A}
$$

On the other hand, as $A=\bigcap_{u \in \mathcal{U}} A_{u}$, according to (2.9), one has

$$
\begin{aligned}
(L, y) \in \bigcup_{u \in \mathcal{U}} \operatorname{epi}\left(F+I_{A_{u}}\right)^{*} & \Longrightarrow \exists u \in \mathcal{U}:(L, y) \in \operatorname{epi}\left(F+I_{A_{u}}\right)^{*} \\
& \Longrightarrow \exists u \in \mathcal{U}: y-L(x)+F(x) \notin-\operatorname{int} K, \forall x \in A_{u} \cap \operatorname{dom} F \\
& \Longrightarrow y-L(x)+F(x) \notin-\operatorname{int} K, \forall x \in A \cap \operatorname{dom} F \\
& \Longrightarrow(L, y) \in \operatorname{epi}\left(F+I_{A}\right)^{*},
\end{aligned}
$$

which means that

$$
\bigcup_{u \in \mathcal{U}} \operatorname{epi}\left(F+I_{A_{u}}\right)^{*} \subset \operatorname{epi}\left(F+I_{A}\right)^{*} .
$$

\footnotetext{
${ }^{2}$ Note that in the first part of the proof of [11, Theorem 4.2] no assumptions on the convexity or closedness of the mappings $F$ and $G$ are needed.
} 
The conclusion now follows from (4.6), (4.7), and (4.8).

Lemma 4.1. Assume that $f \in \Gamma(X)$ and that $C$ is a nonempty closed convex subset of $X$. Assume further that $G_{u}$ is proper, $S$-convex and $S$-epi closed for each $u \in \mathcal{U}$, and that $A \cap \operatorname{dom} f \neq \emptyset$ (where $A$ is given by (4.2) ). Then ${ }^{3}$

$$
\operatorname{epi}\left(f+i_{A}\right)^{*}=\operatorname{clco}\left(\bigcup_{\left(z^{*}, u\right) \in S^{+} \times \mathcal{U}} \operatorname{epi}\left(f+i_{C}+z^{*} \circ G_{u}\right)^{*}\right) \text {. }
$$

Proof. For each $u \in \mathcal{U}$, set $A_{u}=C \cap G_{u}^{-1}(-S)$. Then $A=\bigcap_{u \in \mathcal{U}} A_{u}$ and $\sup _{u \in \mathcal{U}}(f+$ $\left.i_{A_{u}}\right)(x)=\left(f+i_{A}\right)(x)$ for all $x \in X$. As for each $u \in \mathcal{U}, G_{u}$ is proper, $S$-convex and $S$-epi closed and $A \cap \operatorname{dom} f \neq \emptyset$, one has $f+i_{A_{u}} \in \Gamma(X)$ for all $u \in \mathcal{U}$. Now, take $x_{0} \in A \cap \operatorname{dom} f$ (note that $\left.A \cap \operatorname{dom} f \neq \emptyset\right)$, one gets $\sup _{u \in \mathcal{U}}\left(f+i_{A_{u}}\right)\left(x_{0}\right)=\left(f+i_{A}\right)\left(x_{0}\right)<$ $+\infty$. So, according to [18, Lemma 2.2], it holds

$$
\operatorname{epi}\left(f+i_{A}\right)^{*}=\operatorname{clco}\left[\bigcup_{u \in \mathcal{U}} \operatorname{epi}\left(f+i_{A_{u}}\right)^{*}\right] .
$$

On the other hand, for each $u \in \mathcal{U}$, it follows from [6, Theorem 8.2] that

$$
\operatorname{epi}\left(f+i_{A_{u}}\right)^{*}=\mathrm{cl}\left[\bigcup_{z^{*} \in S^{+}} \operatorname{epi}\left(f+i_{C}+z^{*} \circ G_{u}\right)^{*}\right] .
$$

The equality (4.9) now follows from combining (4.10) to (4.11).

We are now in a position to prove the main results of this section. Our purpose is to generalize the representation in Lemma 4.1 to the vector case. The difficulty in such a generalization is that the set epi $\left(F+I_{A}\right)^{*}$ in general is not convex [11, Example 2.6], and hence, it is almost no hope for a representation of the same form as in (4.9). Fortunately, with the help of Proposition 3.3, (4.9) can be generalized with the use of the $k$-sectionally convex hull, as shown in the next theorem.

We need a hypothesis on the convexity of data from (RVP) first.

\begin{tabular}{l|l}
$\left(\mathcal{H}_{0}\right)$ & $F$ is $K$-convex and positively $K$-lsc, $G_{u}: X \rightarrow Z$ is $S$-convex and \\
& $S$-epi-closed for all $u \in \mathcal{U}$, and $C$ is nonempty, closed and convex.
\end{tabular}

Theorem 4.1. Assume that $\left(\mathcal{H}_{0}\right)$ holds. Then, for each $k \in \operatorname{int} K$, one has

$$
\operatorname{epi}\left(F+I_{A}\right)^{*}=\operatorname{cl}\left(\operatorname{sco}_{k} \mathcal{A}_{k}\right) \text {. }
$$

Proof. Take $\bar{k} \in \operatorname{int} K$. By Proposition 4.1, epi $\left(F+I_{A}\right)^{*} \supset \mathcal{A}_{\bar{k}}$. Moreover, by [11, Lemma 3.6] and Proposition 3.3. the set epi $\left(F+I_{A}\right)^{*}$ is closed and $\bar{k}$-sectionally convex, respectively, and so,

$$
\operatorname{epi}\left(F+I_{A}\right)^{*} \supset \operatorname{cl}\left(\operatorname{sco}_{\bar{k}} \mathcal{A}_{\bar{k}}\right)
$$

\footnotetext{
3 This would be an elementary result in the study of robust optimization problems. However, to the surprise of the authors, we could not find it in the references we had in hand and so we insert a short proof here.
} 
We now prove the converse of the inclusion (4.12). For this, take $(\bar{L}, \bar{y}) \in \operatorname{epi}\left(F+I_{A}\right)^{*}$ and we will prove that $(\bar{L}, \bar{y}) \in \operatorname{cl}\left(\operatorname{sco}_{\bar{k}} \mathcal{A}_{\bar{k}}\right)$. Let us structure the rest of our proof in five steps.

- Step 1. Prove that the set $(\bar{L}-F)(A \cap \operatorname{dom} F)-\operatorname{int} K$ is convex. Observe firstly that as $G_{u}$ is $S$-convex for all $u \in \mathcal{U}$, and $C$ is convex, the feasible set $A$ is convex. Also, $F-\bar{L}$ is a $K$-convex mapping. Thus, $(F-\bar{L})(A \cap \operatorname{dom} F)+\operatorname{int} K$ is convex (see [11, Remark 4.1]), and so is $(\bar{L}-F)(A \cap \operatorname{dom} F)-\operatorname{int} K$.

- Step 2. As $(\bar{L}, \bar{y}) \in \operatorname{epi}\left(F+I_{A}\right)^{*}$, it follows from characterizing (2.9) that

$$
\bar{y} \notin(\bar{L}-F)(A \cap \operatorname{dom} F)-\operatorname{int} K \text {. }
$$

So, applying the convex separation theorem [19, Lemma 3.4], there is $y^{*} \in Y^{*} \backslash\left\{0_{Y^{*}}\right\}$ such that

$$
y^{*}(w)<y^{*}(\bar{y}), \quad \forall w \in(\bar{L}-F)(A \cap \operatorname{dom} F)-\operatorname{int} K .
$$

It then follows from [11, Lemma 3.3] that

$$
\begin{gathered}
y^{*} \circ(\bar{L}-F)(x) \leq y^{*}(\bar{y}), \forall x \in A \cap \operatorname{dom} F, \\
y^{*} \in K^{+} \text {and } y^{*}\left(k^{\prime}\right)>0, \forall k^{\prime} \in \operatorname{int} K .
\end{gathered}
$$

- Step 3. It is easy to see that (4.15) is equivalent to $y^{*}(\bar{y}) \geq\left(y^{*} \circ F+i_{A}\right)^{*}\left(y^{*} \circ \bar{L}\right)$, or equivalently, $\left(y^{*} \circ \bar{L}, y^{*}(\bar{y})\right) \in \operatorname{epi}\left(y^{*} \circ F+i_{A}\right)^{*}$. On the other hand, as $y^{*} \in K^{+} \backslash\left\{0_{Y^{*}}\right\}$ and $F$ is $K$-convex and positively $K$-lsc, one has $y^{*} \circ F \in \Gamma(X)$ and now Lemma 4.1, applying to $f=y^{*} \circ F$, gives us

$$
\operatorname{epi}\left(y^{*} \circ F+i_{A}\right)^{*}=\operatorname{clco} \tilde{\mathcal{A}}
$$

where $\tilde{\mathcal{A}}:=\bigcup_{\left(z^{*}, u\right) \in S^{+} \times \mathcal{U}} \operatorname{epi}\left(y^{*} \circ F+i_{C}+z^{*} \circ G_{u}\right)^{*}$. Since $\left(y^{*} \circ \bar{L}, y^{*}(\bar{y})\right) \in \operatorname{epi}\left(y^{*} \circ F+i_{A}\right)^{*}$, it follows from (4.17) that there exist a net $\left(x_{\alpha}^{*}, r_{\alpha}\right)_{\alpha \in D} \subset \operatorname{co} \tilde{\mathcal{A}} \tilde{4}$ such that $\left(x_{\alpha}^{*}, r_{\alpha}\right) \rightarrow$ $\left(y^{*} \circ \bar{L}, y^{*}(\bar{y})\right)$. So, for each $\alpha \in D$, there are a finite index set $I_{\alpha}$, and finite sequences $\left(z_{\alpha_{i}}^{*}\right)_{i \in I_{\alpha}} \subset S^{+},\left(u_{\alpha_{i}}\right)_{i \in I_{\alpha}} \subset \mathcal{U},\left(x_{\alpha_{i}}^{*}\right)_{i \in I_{\alpha}} \subset X^{*},\left(r_{\alpha_{i}}\right)_{i \in I_{\alpha}} \subset \mathbb{R}$ and $\left(\lambda_{\alpha_{i}}\right)_{i \in I_{\alpha}} \subset \mathbb{R}_{+} \backslash\{0\}$ such that $\sum_{i \in I_{\alpha}} \lambda_{\alpha_{i}}=1, \sum_{i \in I_{\alpha}} \lambda_{\alpha_{i}}\left(x_{\alpha_{i}}^{*}, r_{\alpha_{i}}\right)=\left(x_{\alpha}^{*}, r_{\alpha}\right)$, and

$$
\left(x_{\alpha_{i}}^{*}, r_{\alpha_{i}}\right) \in \operatorname{epi}\left(y^{*} \circ F+i_{C}+z_{\alpha_{i}}^{*} \circ G_{u_{\alpha_{i}}}\right), \forall i \in I_{\alpha} .
$$

- Step 4. As $\bar{k} \in \operatorname{int} K$, it follows from (4.16) that $y^{*}(\bar{k})>0$. For each $\alpha \in D$ and $i \in I_{\alpha}$, let us define the elements $y_{\alpha_{i}} \in Y, \tilde{z}_{\alpha_{i}}^{*} \in Z^{*}$, and the mapping $L_{\alpha_{i}}: X \rightarrow Y$, respectively by

$$
y_{\alpha_{i}}:=\bar{y}+\frac{r_{\alpha_{i}}-y^{*}(\bar{y})}{y^{*}(\bar{k})} \bar{k}, \tilde{z}_{\alpha_{i}}^{*}(z):=\frac{z_{\alpha_{i}}^{*}(z)}{y^{*}(\bar{k})}, L_{\alpha_{i}}(x):=\bar{L}(x)+\frac{x_{\alpha_{i}}^{*}(x)-y^{*} \circ \bar{L}(x)}{y^{*}(\bar{k})} \bar{k} .
$$

Then, it is easy to check that

$$
L_{\alpha_{i}} \in \mathcal{L}(X, Y), \tilde{z}_{\alpha_{i}}^{*} \in S^{+}, y^{*}\left(y_{\alpha_{i}}\right)=r_{\alpha_{i}}, y^{*} \circ L_{\alpha_{i}}=x_{\alpha_{i}}^{*}, y^{*} \circ\left(\bar{k} \cdot \tilde{z}_{\alpha_{i}}^{*}\right)=z_{\alpha_{i}}^{*},
$$
and

$$
\left(\sum_{i \in I_{\alpha}} \lambda_{\alpha_{i}} L_{\alpha_{i}}, \sum_{i \in I_{\alpha}} \lambda_{\alpha_{i}} y_{\alpha_{i}}\right) \longrightarrow(\bar{L}, \bar{y}) .
$$

We now show that for each $\alpha \in D$ and $i \in I_{\alpha},\left(L_{\alpha_{i}}, y_{\alpha_{i}}\right) \in \mathcal{A}_{\bar{k}}$. It follows from (4.20) and (4.18) that

$$
y^{*}\left(y_{\alpha_{i}}\right) \geq\left(y^{*} \circ F+i_{C}+y_{\alpha_{i}}^{*} \circ\left(\bar{k} \cdot \tilde{z}_{\alpha_{i}}^{*}\right) \circ G_{u_{\alpha_{i}}}\right)^{*}\left(y^{*} \circ L_{\alpha_{i}}\right),
$$

\footnotetext{
${ }^{4}$ For the sake of simplicity, we write $\left(x_{\alpha}^{*}, r_{\alpha}\right)_{\alpha \in D}$ for $\left(\left(x_{\alpha}^{*}, r_{\alpha}\right)\right)_{\alpha \in D}$.
} 
which is equivalent to

$$
y^{*}\left(y_{\alpha_{i}}\right) \geq y^{*} \circ L_{\alpha_{i}}(x)-y^{*} \circ F(x)-y^{*} \circ\left(\bar{k} \cdot \tilde{z}_{\alpha_{i}}^{*}\right) \circ G_{u_{\alpha_{i}}}(x), \quad \forall x \in C \cap \operatorname{dom} F,
$$

or equivalently,

$$
y^{*}\left[L_{\alpha_{i}}(x)-F(x)-\left(\bar{k} \cdot \tilde{z}_{\alpha_{i}}^{*}\right) \circ G_{u_{\alpha_{i}}}(x)-y_{\alpha_{i}}\right] \leq 0, \quad \forall x \in C \cap \operatorname{dom} F .
$$

The last inequality, together with (4.16), yields

$$
y_{\alpha_{i}} \notin L_{\alpha_{i}}(x)-F(x)-\left(\bar{k} \cdot \tilde{z}_{\alpha_{i}}^{*}\right) \circ G_{u_{\alpha_{i}}}(x)-\operatorname{int} K, \quad \forall x \in C \cap \operatorname{dom} F,
$$

which in turn yields (see (2.9))

$$
\left(L_{\alpha_{i}}, y_{\alpha_{i}}\right) \in \operatorname{epi}\left(F+I_{C}+\left(\bar{k} \cdot \tilde{z}_{\alpha_{i}}^{*}\right) \circ G_{u_{\alpha_{i}}}\right)^{*} \subset \mathcal{A}_{\bar{k}} .
$$

- Step 5. Let $E_{\bar{k}}=\bar{k} \cdot\left(X^{*} \times \mathbb{R}\right)$. According to Proposition 3.1, one has

$$
\operatorname{sco}_{\bar{k}} \mathcal{A}_{\bar{k}}:=\bigcup_{(L, y) \in \mathcal{L}(X, Y) \times Y} \operatorname{co}\left[\mathcal{A}_{\bar{k}} \cap\left(E_{\bar{k}}+(L, y)\right)\right] .
$$

For all $\alpha \in D$, it follows from (4.22) and (4.19) that

$$
\left(L_{\alpha_{i}}, y_{\alpha_{i}}\right) \in \mathcal{A}_{\bar{k}} \cap\left(E_{\bar{k}}+(\bar{L}, \bar{y})\right), \quad \forall i \in I_{\alpha},
$$

and hence, if take $L_{\alpha}:=\sum_{i \in I_{\alpha}} \lambda_{\alpha_{i}} L_{\alpha_{i}}$ and $y_{\alpha}:=\sum_{i \in I_{\alpha}} \lambda_{\alpha_{i}} y_{\alpha_{i}}$ then it holds $\left(L_{\alpha}, y_{\alpha}\right) \in$ $\operatorname{co}\left[\mathcal{A}_{\bar{k}} \cap\left(E_{\bar{k}}+(\bar{L}, \bar{y})\right)\right] \subset \operatorname{sco}_{\bar{k}} \mathcal{A}_{\bar{k}}$. From (4.21), $(\bar{L}, \bar{y})=\lim _{\alpha \in D}\left(L_{\alpha}, y_{\alpha}\right)$, showing that $(\bar{L}, \bar{y}) \in \operatorname{cl}\left(\operatorname{sco}_{\bar{k}} \mathcal{A}_{\bar{k}}\right)$ and we are done.

Corollary 4.1. Assume that $\left(\mathcal{H}_{0}\right)$ holds. Then one has

$$
\operatorname{epi}\left(F+I_{A}\right)^{*}=\operatorname{scl}_{k}\left(\operatorname{sco}_{k} \mathcal{A}_{k}\right), \forall k \in \operatorname{int} K \text {. }
$$

Proof. Indeed, take $\bar{k} \in \operatorname{int} K$, according to Theorem 4.1.

$$
\operatorname{epi}\left(F+I_{A}\right)^{*}=\operatorname{cl}\left(\operatorname{sco}_{\bar{k}} \mathcal{A}\right) \supset \operatorname{scl}_{\bar{k}}\left(\operatorname{sco}_{\bar{k}} \mathcal{A}\right)
$$

(note that all closed subsets are $k$-sectionally closed).

On the other hand, according to Proposition $3.2($ iii $)$, one has

$$
\operatorname{scl}_{\bar{k}}\left(\operatorname{sco}_{\bar{k}} \mathcal{A}_{\bar{k}}\right)=\bigcup_{(L, y) \in \mathcal{L}(X, Y) \times Y} \operatorname{cl} \operatorname{co}\left[\mathcal{A}_{\bar{k}} \cap\left(E_{\bar{k}}+(L, y)\right)\right] .
$$

For each $(\bar{L}, \bar{y})$, we can see from the proof of Theorem 4.1 that $(\bar{L}, \bar{y})$ is the limits of the net $\left(L_{\alpha}, y_{\alpha}\right)_{\alpha \in D}$ with $\left(L_{\alpha}, y_{\alpha}\right) \in \operatorname{co}\left[\mathcal{A}_{\bar{k}} \cap\left(E_{\bar{k}}+(\bar{L}, \bar{y})\right)\right]$ for all $\alpha \in D$. This yields $(\bar{L}, \bar{y}) \subset \operatorname{cl} \operatorname{co}\left[\mathcal{A}_{\bar{k}} \cap\left(E_{\bar{k}}+(\bar{L}, \bar{y})\right)\right] \subset \operatorname{scl}_{\bar{k}}\left(\operatorname{sco}_{\bar{k}} \mathcal{A}_{\bar{k}}\right)$. So,

$$
\operatorname{epi}\left(F+I_{A}\right)^{*} \subset \operatorname{scl}_{\bar{k}}\left(\operatorname{sco}_{\bar{k}} \mathcal{A}_{\bar{k}}\right)
$$

and hence (4.23) follows from (4.24) and (4.25).

Theorem 4.2. Assume that $\left(\mathcal{H}_{0}\right)$ holds. Then, for all $k \in \operatorname{int} K$,

$$
\operatorname{epi}\left(F+I_{A}\right)^{*}=\operatorname{cl}\left(\operatorname{scO}_{k} \mathcal{B}\right)=\operatorname{cl}\left(\operatorname{sco}_{k} \mathcal{A}\right) .
$$

Proof. Take $\bar{k} \in \operatorname{int} K$. It follows from Proposition 4.1 that

$$
\operatorname{epi}\left(F+I_{A}\right)^{*} \supset \mathcal{B} \supset A \supset \mathcal{A}_{\bar{k}}
$$

and as epi $\left(F+I_{A}\right)^{*}$ is $\bar{k}$-sectionally convex (by Proposition 3.3) and closed (see [11, Lemma 3.6]), one gets

$$
\operatorname{epi}\left(F+I_{A}\right)^{*} \supset \operatorname{cl}\left(\operatorname{sco}_{k} \mathcal{B}\right) \supset \operatorname{cl}\left(\operatorname{scO}_{k} \mathcal{A}\right) \supset \operatorname{cl}\left(\operatorname{sco}_{k} \mathcal{A}_{\bar{k}}\right) .
$$


On the other hand, under the assumption $\left(\mathcal{H}_{0}\right)$, Theorem 4.1 gives that epi $\left(F+I_{A}\right)^{*}=$ $\operatorname{cl}\left(\operatorname{sco}_{\bar{k}} \mathcal{A}_{\bar{k}}\right)$, and hence, (4.26) follows.

In the case with the absence of the uncertainty, i.e., the uncertainty set $\mathcal{U}$ is a singleton, Theorems 4.1-4.2 collapse to the ones that cover both Theorems 4.1 and 4.2 in [11].

Corollary 4.2. Assume that $F: X \rightarrow Y^{\bullet}$ is a proper $K$-convex and positively $K$ lsc mapping, that $G: X \rightarrow Z$ is a proper $S$-convex and $S$-epi closed mapping, and that $C$ is nonempty, closed and convex. Assume further that $B \cap \operatorname{dom} F \neq \emptyset$ where $B:=C \cap G^{-1}(-S)$. Then

$$
\begin{aligned}
\operatorname{epi}\left(F+I_{B}\right)^{*} & =\operatorname{cl}\left(\bigcup_{T \in \mathcal{L}_{+}(S, K)} \operatorname{epi}\left(F+I_{C}+T \circ G\right)^{*}\right) \\
& =\operatorname{cl}\left(\bigcup_{T \in \mathcal{L}_{+}^{w}(S, K)} \bigcap_{v \in I_{-S}^{*}(T)}\left[\operatorname{epi}\left(F+I_{C}+T \circ G\right)^{*}+\left(0_{\mathcal{L}}, v\right)\right]\right) \\
& =\operatorname{cl}\left(\bigcup_{z^{*} \in S^{+}} \operatorname{epi}\left(F+I_{C}+\left(\bar{k} \cdot z^{*}\right) \circ G\right)^{*}\right) .
\end{aligned}
$$

Proof. Let $\bar{k} \in \operatorname{int} K$. In the case where the uncertainty set $\mathcal{U}$ is a singleton, the qualifying sets $\mathcal{A}, \mathcal{A}_{\bar{k}}, \mathcal{B}$ become the following sets, respectively

$$
\begin{aligned}
\tilde{\mathcal{A}} & =\bigcup_{T \in \mathcal{L}_{+}(S, K)} \operatorname{epi}\left(F+I_{C}+T \circ G\right)^{*}, \\
\tilde{\mathcal{A}}_{\bar{k}} & =\bigcup_{z^{*} \in S^{+}} \operatorname{epi}\left(F+I_{C}+\left(\bar{k} \cdot z^{*}\right) \circ G\right)^{*}, \\
\tilde{\mathcal{B}} & =\bigcup_{T \in \mathcal{L}_{+}^{w}(S, K)} \bigcap_{v \in I_{-S}^{*}(T)}\left[\operatorname{epi}\left(F+I_{C}+T \circ G\right)^{*}+\left(0_{\mathcal{L}}, v\right)\right] .
\end{aligned}
$$

In such a case $\left(\mathcal{U}\right.$ is a singleton) Proposition 3.3 gives $\tilde{\mathcal{A}}_{\bar{k}} \subset \tilde{\mathcal{A}} \subset \tilde{\mathcal{B}} \subset \operatorname{epi}\left(F+I_{B}\right)^{*}$, which, together with the fact that $\operatorname{epi}\left(F+I_{B}\right)^{*}$ is closed (see [11, Lemma 3.6]), leads to

$$
\operatorname{cl} \tilde{\mathcal{A}}_{\bar{k}} \subset \operatorname{cl} \tilde{\mathcal{A}} \subset \operatorname{cl} \tilde{\mathcal{B}} \subset \operatorname{epi}\left(F+I_{B}\right)^{*} .
$$

On the other hand, according to Proposition 3.4, the set $\tilde{\mathcal{A}}_{\bar{k}}$ is $\bar{k}$-sectionally convex, and hence, $\operatorname{sco}_{\bar{k}} \tilde{\mathcal{A}}_{\bar{k}}=\tilde{\mathcal{A}}_{\bar{k}}$. Now, Theorem 4.1 yields epi $\left(F+I_{B}\right)^{*}=\operatorname{cl}\left(\operatorname{sco}_{\bar{k}} \tilde{\mathcal{A}}_{\bar{k}}\right)=\operatorname{cl} \tilde{\mathcal{A}}_{\bar{k}}$, which, together with (4.27) leads to

$$
\operatorname{cl} \tilde{\mathcal{A}}_{\bar{k}} \subset \operatorname{cl} \tilde{\mathcal{A}} \subset \operatorname{cl} \tilde{\mathcal{B}} \subset \operatorname{epi}\left(F+I_{B}\right)^{*} \subset \operatorname{cl} \tilde{\mathcal{A}}_{\bar{k}},
$$

and the conclusion follows.

\section{Robust vector Farkas-type results}

We retain all the notations used in the previous sections and consider the robust vector optimization problem (RVP) defined by (4.1) with its feasible set $A$ as in (4.2) and the 
assumption $A \cap \operatorname{dom} F \neq \emptyset$. Consider the qualifying sets $\mathcal{A}, \mathcal{B}$ and $\mathcal{A}_{k}$ (for some $k \in$ int $K$ ) defined respectively by (4.3), (4.4), and (4.5). Moreover, we say that $\mathcal{A}\left(\mathcal{B}, \mathcal{A}_{k}\right.$, respectively) is $k$-sectionally convex and closed regarding $\mathcal{V} \times \mathcal{W}$ if $\operatorname{cl}\left(\operatorname{sco}_{k} \mathcal{A}\right) \cap(\mathcal{V} \times \mathcal{W})=$ $\mathcal{A} \cap(\mathcal{V} \times \mathcal{W})\left(\operatorname{cl}\left(\operatorname{sco}_{k} \mathcal{B}\right) \cap(\mathcal{V} \times \mathcal{W})=\mathcal{B} \cap(\mathcal{V} \times \mathcal{W}), \operatorname{cl}\left(\operatorname{sco}_{k} \mathcal{A}_{k}\right) \cap(\mathcal{V} \times \mathcal{W})=\mathcal{A}_{k} \cap(\mathcal{V} \times \mathcal{W})\right.$ respectively). Let $\emptyset \neq \mathcal{V} \subset \mathcal{L}(X, Y)$ and $\emptyset \neq \mathcal{W} \subset Y$.

We now establish some principles and results on $(\mathcal{V}, \mathcal{W})$-stable Farkas lemma for vector-valued systems concerning the robust vector optimization problem (RVP). In the first one, Theorem 5.1, for the sake of completeness, we quote $[(\mathrm{a}) \Leftrightarrow(\mathrm{b})]$ from $[9$, Theorem 3.2(ii)]. Note also that Theorem 5.1 extends [10, Theorems 1,2].

Theorem 5.1 (Principles of stable robust vector Farkas lemma I). Consider the following statements

(a) $\operatorname{epi}\left(F+I_{A}\right)^{*} \cap(\mathcal{V} \times \mathcal{W})=\mathcal{A} \cap(\mathcal{V} \times \mathcal{W})$,

(b) $\operatorname{epi}\left(F+I_{A}\right)^{*} \cap(\mathcal{V} \times \mathcal{W})=\mathcal{B} \cap(\mathcal{V} \times \mathcal{W})$,

(c) For any $(L, y) \in \mathcal{V} \times \mathcal{W}$, the next two assertions are equivalent:

$(\alpha) G_{u}(x) \in-S, x \in C, \forall u \in \mathcal{U} \Longrightarrow y-L(x)+F(x) \notin-\operatorname{int} K$,

( $\beta)$ There exist $u \in \mathcal{U}$ and $T \in \mathcal{L}_{+}(S, K)$ such that

$$
F(x)+T \circ G_{u}(x)-L(x)+y \notin-\operatorname{int} K, \forall x \in C .
$$

(d) For any $(L, y) \in \mathcal{V} \times \mathcal{W}$, the next two assertions are equivalent:

$(\alpha) G_{u}(x) \in-S, x \in C, \forall u \in \mathcal{U} \Longrightarrow y-L(x)+F(x) \notin-\operatorname{int} K$,

$(\gamma)$ There exist $u \in \mathcal{U}$ and $T \in \mathcal{L}_{+}^{w}(S, K)$ such that

$$
F(x)+T \circ G_{u}(x)-L(x)+y \notin-T(S)-\operatorname{int} K, \forall x \in C .
$$

Then, $[(\mathrm{a}) \Leftrightarrow(\mathrm{c})]$ and $[(\mathrm{b}) \Leftrightarrow(\mathrm{d})]$.

Proof. The first equivalence, $[(\mathrm{a}) \Leftrightarrow(\mathrm{c})]$, is Theorem 3.2(ii) in [9]. However, for the sake of completeness, we give briefly the proof here. It is easy to see that $(\alpha)$ is equivalent to $(L, y) \in \operatorname{epi}\left(F+I_{A}\right)^{*}$ while $(\beta)$ is equivalent to $(L, y) \in \mathcal{A}$. So, $[(\mathrm{a}) \Leftrightarrow(\mathrm{c})]$ holds. The proof of the second one, $[(\mathrm{b}) \Leftrightarrow(\mathrm{d})]$, can be obtained by using a similar way using the weak cone of positive operators $\mathcal{L}_{+}^{w}(S, K)$ instead of $\mathcal{L}_{+}(S, K)$.

For each $k \in \operatorname{int} K$, recall that (see (4.5))

$$
\mathcal{A}_{k}=\bigcup_{\left(z^{*}, u\right) \in S^{+} \times \mathcal{U}} \operatorname{epi}\left(F+I_{C}+\left(k \cdot z^{*}\right) \circ G_{u}\right)^{*} .
$$

Another principle for stable robust vector Farkas lemma based on $\mathcal{A}_{k}$ is given in the next theorem.

Theorem 5.2 (Principle of stable robust vector Farkas lemma II). Let $k \in \operatorname{int} K$, the following statements are equivelent

$\left(\mathrm{a}_{k}\right) \operatorname{epi}\left(F+I_{A}\right)^{*} \cap(\mathcal{V} \times \mathcal{W})=\mathcal{A}_{k} \cap(\mathcal{V} \times \mathcal{W})$

$\left(c_{k}\right)$ For any $(L, y) \in \mathcal{V} \times \mathcal{W}$, the next two assertions are equivalent:

$(\alpha) G_{u}(x) \in-S, x \in C, \forall u \in \mathcal{U} \Longrightarrow y-L(x)+F(x) \notin-\operatorname{int} K$,

$(\delta)$ There exist $u \in \mathcal{U}$ and $z^{*} \in S^{+}$such that

$$
F(x)+\left(z^{*} \circ G_{u}\right)(x) k-L(x)+y \notin-\operatorname{int} K, \forall x \in C .
$$


Proof. The proof is similar to that of Theorem 5.1. It is clear that $(\alpha)$ is equivalent to $(L, y) \in \operatorname{epi}\left(F+I_{A}\right)^{*}($ by (2.9) $)$ and $(\delta)$ is equivalent to $(L, y) \in \mathcal{A}_{k}$. So, $\left(\mathrm{a}_{k}\right)$ and $\left(\mathrm{c}_{k}\right)$ are equivalent.

Now, we are ready to establish principles of $(\mathcal{V}, \mathcal{W})$-stable robust vector Farkas lemma in convex setting (i.e., under the hypothesis $\left.\left(\mathcal{H}_{0}\right)\right)$. These results are obtained by combining Theorem 5.1 and the results on representations of epi $\left(F+I_{A}\right)^{*}$ provided in Section 4.

Theorem 5.3 (Principles of stable robust convex vector Farkas lemma I). Assume that that the hypothesis $\left(\mathcal{H}_{0}\right)$ holds. Consider the following statements:

$\left(\mathrm{a}_{1}\right) \exists k \in \operatorname{int} K$ s.t. $\mathcal{A}$ is $k$-sectionally convex and closed regarding $\mathcal{V} \times \mathcal{W}$,

$\left(\mathrm{b}_{1}\right) \exists k \in \operatorname{int} K$ s.t. $\mathcal{B}$ is $k$-sectionally convex and closed regarding $\mathcal{V} \times \mathcal{W}$.

Then, $\left[\left(\mathrm{a}_{1}\right) \Leftrightarrow(\mathrm{c})\right]$ and $\left[\left(\mathrm{b}_{1}\right) \Leftrightarrow(\mathrm{d})\right]$, where $(\mathrm{c})$ and $(\mathrm{d})$ are the statements in Theorem 5.1.

Proof. Let $k \in \operatorname{int} K$. As $\left(\mathcal{H}_{0}\right)$ holds, it follows from Theorem 4.2 that

$$
\operatorname{epi}\left(F+I_{A}\right)^{*}=\operatorname{cl}\left(\operatorname{sco}_{k} \mathcal{A}\right)=\operatorname{cl}\left(\operatorname{sco}_{k} \mathcal{B}\right) .
$$

So, the statements $\left(\mathrm{a}_{1}\right)$ and $\left(\mathrm{b}_{1}\right)$ are respectively equivalent to $\operatorname{epi}\left(F+I_{A}\right)^{*} \cap(\mathcal{V} \times \mathcal{W})=\mathcal{A} \cap(\mathcal{V} \times \mathcal{W})$ and $\operatorname{epi}\left(F+I_{A}\right)^{*} \cap(\mathcal{V} \times \mathcal{W})=\mathcal{B} \cap(\mathcal{V} \times \mathcal{W})$ for any $\emptyset \neq \mathcal{V} \subset \mathcal{L}(X, Y)$ and any $\emptyset \neq \mathcal{W} \subset Y$. The conclusion now follows from Theorem 5.1.

For each $k \in \operatorname{int} K$, recall that the set $\mathcal{A}_{k}$ is defined by (4.5). We are now seeking for other alternative qualifying conditions based on the set $\mathcal{A}_{k}$ that guarantee the previous versions of robust vector Farkas lemmas.

Theorem 5.4 (Stable robust convex vector Farkas lemma I). Assume that the hypothesis $\left(\mathcal{H}_{0}\right)$ and the following condition $\left(\mathrm{a}_{2}\right)$ hold:

$\left(\mathrm{a}_{2}\right) \exists k \in \operatorname{int} K$ s.t. $\mathcal{A}_{k}$ is $k$-sectionally convex and closed regarding $\mathcal{V} \times \mathcal{W}$.

Then, the assertions (c), (d) in Theorem 5.1 hold.

Proof. Firstly, according to Proposition 4.1, one has

$$
\mathcal{A}_{k} \cap(\mathcal{V} \times \mathcal{W}) \subset \mathcal{A} \cap(\mathcal{V} \times \mathcal{W}) \subset \mathcal{B} \cap(\mathcal{V} \times \mathcal{W}) \subset \operatorname{epi}\left(F+I_{A}\right)^{*} \cap(\mathcal{V} \times \mathcal{W}) .
$$

On the other hand, under the hypothesis $\left(\mathcal{H}_{0}\right)$, Theorem 4.1 yields epi $\left(F+I_{A}\right)^{*}=$ $\mathrm{cl}\left(\mathrm{sco}_{k} \mathcal{A}_{k}\right)$, which combining with $\left(\mathrm{a}_{2}\right)$, one gets

$$
\operatorname{epi}\left(F+I_{A}\right)^{*} \cap(\mathcal{V} \times \mathcal{W})=\operatorname{cl}\left(\operatorname{sco}_{k_{0}} \mathcal{A}_{k_{0}}\right) \cap(\mathcal{V} \times \mathcal{W})=\mathcal{A}_{k_{0}} \cap(\mathcal{V} \times \mathcal{W}),
$$

This, together with (5.1), assures that (a) and (b) in Theorem 5.1 hold. The conclusion now follows from Theorem 5.1 .

Theorem 5.5 (Principles for stable robust convex vector Farkas-lemma II). Let $k \in$ int $K$ and assume that the hypothesis $\left(\mathcal{H}_{0}\right)$ holds. Then the following statements $\left(\mathrm{a}_{k}^{\prime}\right)$ and $\left(\mathrm{c}_{k}\right)$ are equivalent:

$\left(\mathrm{a}_{k}^{\prime}\right) \mathcal{A}_{k}$ is $k$-sectionally convex and closed regarding $\mathcal{V} \times \mathcal{W}$, 
$\left(c_{k}\right)$ For any $(L, y) \in \mathcal{V} \times \mathcal{W}$, the next two assertions are equivalent:

$(\alpha) G_{u}(x) \in-S, x \in C, \forall u \in \mathcal{U} \Longrightarrow y-L(x)+F(x) \notin-\operatorname{int} K$,

$(\delta)$ There exist $u \in \mathcal{U}$ and $z^{*} \in S^{+}$such that

$$
F(x)+\left(z^{*} \circ G_{u}\right)(x) k-L(x)+y \notin-\operatorname{int} K, \forall x \in C .
$$

Proof. As $\left(\mathcal{H}_{0}\right)$ holds, it follows from Theorem 4.1 that epi $\left(F+I_{A}\right)^{*}=\operatorname{cl}\left(\operatorname{sco}_{k} \mathcal{A}_{k}\right)$, and then $\left(\mathrm{a}_{k}^{\prime}\right)$ is nothing else but $\left(\mathrm{a}_{k}\right)$ in Theorem 5.2. The conclusion now follows from Theorem 5.2.

Some sufficient conditions for $k$-sectional convexity and $k$-sectional closedness of the sets $\mathcal{A}_{k}$ (with $k \in \operatorname{int} K$ ), $\mathcal{A}$, and $\mathcal{B}$ will be given below. We first consider some more assumptions:

$\left(\mathcal{H}_{1}\right) \mid$ The collection $\left(u \mapsto G_{u}(x)\right)_{x \in C \cap \operatorname{dom} F}$ is uniformly $S^{+}$-concave,

$\left(\mathcal{H}_{2}\right) \mid$ The mapping $u \mapsto G_{u}(x)$ is $S^{+}$-uniformly usc for each $x \in C \cap \operatorname{dom} F$.

Proposition 5.1. If $\left(\mathcal{H}_{0}\right)$ and $\left(\mathcal{H}_{1}\right)$ hold then $\mathcal{A}_{k}$ is $k$-sectionally convex for each $k \in \operatorname{int} K$.

Proof. Take $k_{0} \in \operatorname{int} K$ and $\left(L_{0}, y_{0}\right) \in \mathcal{L}(X, Y) \times Y$, we will prove that $\mathcal{A}_{k_{0}} \cap\left[E_{k_{0}}+\right.$ $\left.\left(L_{0}, y_{0}\right)\right]$ is a convex set with $E_{k_{0}}=k_{0} \cdot\left(X^{*} \times \mathbb{R}\right)$. For this, take $a_{1}, a_{2} \in \mathcal{A}_{k_{0}} \cap\left[E_{k_{0}}+\right.$ $\left.\left(L_{0}, y_{0}\right)\right]$ and $\left.\lambda \in\right] 0,1\left[\right.$, we show that $\lambda a_{1}+(1-\lambda) a_{2} \in \mathcal{A}_{k_{0}}$.

For $i=1,2$, as $a_{i} \in \mathcal{A}_{k_{0}} \cap\left[E_{k_{0}}+\left(L_{0}, y_{0}\right)\right]$, there exist $\left(x_{i}^{*}, r_{i}\right) \in X^{*} \times \mathbb{R}$ and $\left(z_{i}^{*}, u_{i}\right) \in$ $S^{+} \times \mathcal{U}$ such that $a_{i}=k_{0}\left(x_{i}^{*}, r_{i}\right)+\left(L_{0}, y_{0}\right)$ and (according to (2.9))

$y_{0}+r_{i} k_{0}-L_{0}(x)-\left\langle x_{i}^{*}, x\right\rangle k_{0}+F(x)+\left(z_{i}^{*} \circ G_{u_{i}}\right)(x) k_{0} \notin-\operatorname{int} K, \forall x \in C \cap \operatorname{dom} F$.

Lemma 2.1 applying to $y=y_{0}-L_{0}(x)+F(x), \alpha=r_{1}-\left\langle x_{1}^{*}, x\right\rangle+\left(z_{1}^{*} \circ G_{u_{1}}\right)(x)$ and $\beta=r_{2}-\left\langle x_{2}^{*}, x\right\rangle+\left(z_{2}^{*} \circ G_{u_{2}}\right)(x)$ yields

$$
y_{0}+\left[\lambda r_{1}+(1-\lambda) r_{2}\right] k_{0}-L_{0}(x)-\left\langle\lambda x_{1}^{*}+(1-\lambda) x_{2}^{*}, x\right\rangle k_{0}+F(x)
$$

$+\left[\lambda\left(z_{1}^{*} \circ G_{u_{1}}\right)(x)+(1-\lambda)\left(z_{2}^{*} \circ G_{u_{2}}\right)(x)\right] k_{0} \notin-\operatorname{int} K, \quad \forall x \in C \cap \operatorname{dom} F$.

On the other hand, as $\left(\mathcal{H}_{1}\right)$ holds, there exists $\left(\bar{z}^{*}, \bar{u}\right) \in S^{+} \times \mathcal{U}$ such that

$$
\lambda\left(z_{1}^{*} \circ G_{u_{1}}\right)(x)+(1-\lambda)\left(z_{2}^{*} G_{u_{2}}\right)(x) \leq\left(\bar{z}^{*} \circ G_{\bar{u}}\right)(x), \forall x \in C \cap \operatorname{dom} F,
$$

or, equivalently,

$$
\left(\left(\bar{z}^{*} \circ G_{\bar{u}}\right)(x)-\left[\lambda\left(z_{1}^{*} \circ G_{u_{1}}\right)(x)+(1-\lambda)\left(z_{2}^{*} G_{u_{2}}\right)(x)\right]\right) k_{0} \in K .
$$

By (2.1), we get from (5.2) and (5.3),

$y_{0}+\left[\lambda r_{1}+(1-\lambda) r_{2}\right] k_{0}-L_{0}(x)-\left\langle\lambda x_{1}^{*}+(1-\lambda) x_{2}^{*}, x\right\rangle k_{0}+F(x)+\left(\bar{z}^{*} \circ G_{\bar{u}}\right)(x) k_{0} \notin-\operatorname{int} K$, for all $x \in C \cap \operatorname{dom} F$, which means that (see (2.9) $) \lambda a_{1}+(1-\lambda) a_{2}=\left(L_{0}, y_{0}\right)+$ $k_{0}\left[\lambda\left(x_{1}^{*}, r_{1}\right)+(1-\lambda)\left(x_{2}^{*}, r_{2}\right)\right] \in \operatorname{epi}\left(F+\left(k_{0} \cdot \bar{z}^{*}\right) \circ G\right)^{*} \subset \mathcal{A}_{k_{0}}$. The proof is complete.

Proposition 5.2. Assume that $\mathcal{U}$ is a compact space, that $Z$ is a normed space, and that $\left(\mathcal{H}_{2}\right)$ and the following Slater-type condition hold:

$\left(C_{0}\right) \forall u \in \mathcal{U}, \exists x_{u} \in C \cap \operatorname{dom} F: G_{u}\left(x_{u}\right) \in-\operatorname{int} S$.

Then, $\mathcal{A}_{k}$ is k-sectionally closed for each $k \in \operatorname{int} K$ 
Proof. Take arbitrarily $k_{0} \in \operatorname{int} K$ and $\left(L_{0}, y_{0}\right) \in \mathcal{L}(X, Y) \times Y$, we will prove that $\mathcal{A}_{k_{0}} \cap\left(E_{k_{0}}+\left(L_{0}, y_{0}\right)\right)$ is closed, where $E_{k_{0}}=k_{0} \cdot\left(X^{*} \times \mathbb{R}\right)$ (defined in (3.10)). For this, take $\left(L_{\alpha}, y_{\alpha}\right)_{\alpha \in D} \subset \mathcal{A}_{k_{0}} \cap\left(E_{k_{0}}+\left(L_{0}, y_{0}\right)\right)$ such that $\left(L_{\alpha}, y_{\alpha}\right) \rightarrow(L, y)$, we need to show that $(L, y) \in \mathcal{A}_{k_{0}} \cap\left(E_{k_{0}}+\left(L_{0}, y_{0}\right)\right)$.

- Firstly, for all $\alpha \in D$, as $\left(L_{\alpha}, y_{\alpha}\right) \in \mathcal{A}_{k_{0}} \cap\left(E_{k_{0}}+\left(L_{0}, y_{0}\right)\right)$, there exist $\left(z_{\alpha}^{*}, u_{\alpha}\right) \in$ $S^{+} \times \mathcal{U}$ and $\left(x_{\alpha}^{*}, r_{\alpha}\right) \in X^{*} \times \mathbb{R}$ such that $\left(L_{\alpha}, y_{\alpha}\right) \in \operatorname{epi}\left(F+I_{C}+\left(k_{0} \cdot z_{\alpha}^{*}\right) \circ G_{u_{\alpha}}\right)^{*}$ and $\left(L_{\alpha}, y_{\alpha}\right)=k_{0}\left(x_{\alpha}^{*}, r_{\alpha}\right)+\left(L_{0}, y_{0}\right)$. Then, by (2.9) $)$ it holds

$$
y_{0}+r_{\alpha} k_{0}-L_{0}(x)-\left\langle x_{\alpha}^{*}, x\right\rangle k_{0}+F(x)+\left(z_{\alpha}^{*} \circ G_{u_{\alpha}}\right)(x) k_{0} \notin-\operatorname{int} K, \forall x \in C \cap \operatorname{dom} F,
$$

or equivalently,

$$
y_{0}-L_{0}(x)+F(x)+\left(r_{\alpha}-\left\langle x_{\alpha}^{*}, x\right\rangle+\left(z_{\alpha}^{*} \circ G_{u_{\alpha}}\right)(x)\right) k_{0} \notin-\operatorname{int} K, \forall x \in C \cap \operatorname{dom} F,
$$

- Next, for each $x \in C \cap \operatorname{dom} F$, according to Lemma 2.2, there is $\alpha_{x} \in \mathbb{R}$ such that

$$
\alpha<\alpha_{x} \Longleftrightarrow y_{0}-L_{0}(x)+F(x)+\alpha k_{0} \in-\operatorname{int} K \text {. }
$$

It follows from (5.5) and (5.4) that

$$
r_{\alpha}-\left\langle x_{\alpha}^{*}, x\right\rangle+\left(z_{\alpha}^{*} \circ G_{u_{\alpha}}\right)(x) \geq \alpha_{x}, \forall x \in C \cap \operatorname{dom} F .
$$

- We now prove that the net $\left(\left\|z_{\alpha}^{*}\right\|\right)_{\alpha \in D}$ is bounded, where $\left\|z_{\alpha}^{*}\right\|:=\sup _{\|z\| \leq 1}\left\langle z_{\alpha}^{*}, z\right\rangle$. Let assume by contradiction that $\left\|z_{\alpha}^{*}\right\| \rightarrow+\infty$. Without loss of generality we can assume that $\left\|z_{\alpha}^{*}\right\|>0$ for all $\alpha \in D$, and hence, according to (5.6),

$$
\left(\tilde{z}_{\alpha}^{*} \circ G_{u_{\alpha}}\right)(x) \geq \frac{1}{\left\|z_{\alpha}^{*}\right\|}\left(\alpha_{x}+\left\langle x_{\alpha}^{*}, x\right\rangle-r_{\alpha}\right), \forall x \in C \cap \operatorname{dom} F, \forall \alpha \in D,
$$

where $\tilde{z}_{\alpha}^{*}=\frac{1}{\left\|z_{\alpha}^{*}\right\|} z_{\alpha}^{*}$.

Note that $\left(L_{\alpha}, y_{\alpha}\right)=k_{0}\left(x_{\alpha}^{*}, r_{\alpha}\right)+\left(L_{0}, y_{0}\right) \rightarrow(L, y)$. We now prove that there is $\left(\bar{x}^{*}, \bar{r}\right) \in X^{*} \times \mathbb{R}$ such that

$$
\left(x_{\alpha}^{*}, r_{\alpha}\right) \rightarrow\left(\bar{x}^{*}, \bar{r}\right) \text { and }(L, y)=k_{0}\left(\bar{x}^{*}, \bar{r}\right)+\left(L_{0}, y_{0}\right)
$$

Indeed, take $\bar{y}^{*} \in Y^{*}$ such that $\bar{y}^{*}\left(k_{0}\right)=1$ (it is possible as $k_{0} \neq 0_{Y}$ ). As $\left(L_{\alpha}, y_{\alpha}\right)=$ $k_{0}\left(x_{\alpha}^{*}, r_{\alpha}\right)+\left(L_{0}, y_{0}\right) \rightarrow(L, y)$, one has $k_{0}\left(x_{\alpha}^{*}, r_{\alpha}\right) \rightarrow\left(L-L_{0}, y-y_{0}\right)$ or equivalently, $k_{0} \cdot x_{\alpha}^{*} \rightarrow L-L_{0}$ and $r_{\alpha} k_{0} \rightarrow y-y_{0}$. Apply $\bar{y}^{*}$ to these expressions, one gets

$$
\begin{aligned}
& \bar{y}^{*} \circ\left(k_{0} \cdot x_{\alpha}^{*}\right)=\left(\bar{y}^{*}\left(k_{0}\right)\right) x_{\alpha}^{*}=x_{\alpha}^{*} \stackrel{*}{\rightarrow} \bar{y}^{*} \circ\left(L-L_{0}\right)=: \bar{x}^{*} \\
& \bar{y}^{*} \circ\left(k_{0} r_{\alpha}\right)=\left(\bar{y}^{*}\left(k_{0}\right) r_{\alpha}=r_{\alpha} \rightarrow \bar{y}^{*}\left(y-y_{0}\right)=: \bar{r},\right.
\end{aligned}
$$

which gives $\left(L_{\alpha}, y_{\alpha}\right)=k_{0}\left(x_{\alpha}^{*}, r_{\alpha}\right)+\left(L_{0}, y_{0}\right) \rightarrow k_{0}\left(\bar{x}^{*}, \bar{r}\right)+\left(L_{0}, y_{0}\right)$ and (5.8) follows by the uniqueness of the limit.

On the other hand, as $\left\|\tilde{z}_{\alpha}^{*}\right\|=1$ for all $\alpha \in D$ by Banach-Alaoglu theorem, without loss of generality, we can assume that $\tilde{z}_{\alpha}^{*} \stackrel{*}{\rightarrow} \tilde{z}^{*} \in Z^{*}$ and as $\mathcal{U}$ is compact we also can assume (without loss of generality) that $u_{\alpha} \rightarrow \bar{u} \in \mathcal{U}$. So, pass to the limit (with $\alpha \in D$ ) in (5.7), taking into account that $\left\|z_{\alpha}^{*}\right\| \rightarrow+\infty$ and that $u \mapsto G_{u}$ is $S^{+}$-uniformly usc for all $x \in C \cap \operatorname{dom} F$ (by $\left(\mathcal{H}_{2}\right)$ ), one gets

$$
\left(\tilde{z}^{*} \circ G_{\bar{u}}\right)(x) \geq 0, \forall x \in C \cap \operatorname{dom} F \text {. }
$$

Next, as $\tilde{z}_{\alpha}^{*} \in S^{+},\left\|\tilde{z}_{\alpha}^{*}\right\|=1$ for all $\alpha \in D$, and $\tilde{z}_{\alpha}^{*} \stackrel{*}{\rightarrow} \tilde{z}^{*}$, it holds $\tilde{z}^{*} \in S^{+} \backslash\left\{0_{Z^{*}}\right\}$. Consequently, $\tilde{z}^{*}(s)>0$ for all $s \in \operatorname{int} S$ which, together with (5.9), yields

$$
G_{\bar{u}}(x) \notin-\operatorname{int} S, \forall x \in C \cap \operatorname{dom} F .
$$


This contradicts $\left(C_{0}\right)$, and hence, the net $\left(\left\|z_{\alpha}^{*}\right\|\right)_{\alpha \in D}$ is bounded.

- As $\left(\left\|z_{\alpha}^{*}\right\|\right)_{\alpha \in D}$ is bounded, we can assume that $z_{\alpha}^{*} \stackrel{*}{\rightarrow} \bar{z}^{*} \in S^{+}$. For each $x \in$ $C \cap \operatorname{dom} F$, pass to the limit in (5.6), with the noting that $u_{\alpha} \rightarrow \bar{u} \in \mathcal{U}, r_{\alpha} \rightarrow \bar{r}$, $x_{\alpha}^{*} \stackrel{*}{\rightarrow} \bar{x}^{*}$, and that $u \mapsto G_{u}$ is $S^{+}$-uniformly usc, one gets

$$
\bar{r}-\left\langle\bar{x}^{*}, x\right\rangle+\left(\bar{z}^{*} \circ G_{\bar{u}}\right)(x) \geq \alpha_{x} .
$$

This, together with (5.5), accounts for

$$
y_{0}-L_{0}(x)+F(x)+\left[\bar{r}-\left\langle\bar{x}^{*}, x\right\rangle+\left(\bar{z}^{*} \circ G_{\bar{u}}\right)(x)\right] k_{0} \notin-\operatorname{int} K,
$$

So, $(L, y)=k_{0}\left(\bar{x}^{*}, \bar{r}\right)+\left(L_{0}, y_{0}\right) \in \operatorname{epi}\left(F+I_{C}+\left(\bar{z}^{*} \circ G_{\bar{u}}\right)\right)^{*} \subset \mathcal{A}_{k_{0}}$, and hence, $(L, y) \in \mathcal{A}_{k_{0}} \cap\left(E_{k_{0}}+\left(L_{0}, y_{0}\right)\right)$. The proof is complete.

Proposition 5.3. Assume that $\mathcal{U}$ is a compact space, that $Z$ is a normed space, and that the hypotheses $\left(\mathcal{H}_{0}\right),\left(\mathcal{H}_{1}\right),\left(\mathcal{H}_{2}\right)$, and the Slater-type condition $\left(C_{0}\right)$ hold. Then the sets $\mathcal{A}_{k}$ (for any $k \in \operatorname{int} K$ ), $\mathcal{A}, \mathcal{B}$ are $k$-sectionally convex and closed.

Proof. Take $k_{0} \in \operatorname{int} K$. According to Corollary 4.1, epi $\left(F+I_{A}\right)^{*}=\operatorname{scl}_{k_{0}}\left(\operatorname{sco}_{k_{0}} \mathcal{A}_{k_{0}}\right)$. Moreover, it follows from Propositions 5.1, 5.2 that $\mathcal{A}_{k_{0}}$ is $k_{0}$-sectionally convex and $k_{0}$-sectionally closed, and hence, $\operatorname{epi}\left(F+I_{A}\right)^{*}=\mathcal{A}_{k_{0}}$. On the other hand, according to Proposition 4.1, epi $\left(F+I_{A}\right)^{*} \supset \mathcal{B} \subset \mathcal{A} \supset \mathcal{A}_{k_{0}}$. So, epi $\left(F+I_{A}\right)^{*}=\mathcal{B}=\mathcal{A}=\mathcal{A}_{k_{0}}$. The conclusion now follows from this and the fact that epi $\left(F+I_{A}\right)^{*}$ is closed and $k$-sectionally convex.

Combining Theorems 5.155.5 and Propositions 5.155.3, we get the following version of stable robust vector Farkas lemma in convex setting.

Theorem 5.6 (Stable robust convex vector Farkas lemma II). Assume that $\mathcal{U}$ is a compact space and $Z$ is a normed space. Assume further that the hypotheses $\left(\mathcal{H}_{0}\right)$, $\left(\mathcal{H}_{1}\right),\left(\mathcal{H}_{2}\right)$, and the Slater-type condition $\left(C_{0}\right)$ hold.

Then, for all $\mathcal{V} \times \mathcal{W} \subset \mathcal{L}(X, Y) \times Y$, the three versions of $\mathcal{V} \times \mathcal{W}$-stable robust Farkas lemma described in (c) and (d) in Theorem 5.1, and $\left(\mathrm{c}_{k}\right)$ (for arbitrary $k \in \operatorname{int} K$ ) in Theorem 5.5 hold.

Proof. Take $\mathcal{V} \times \mathcal{W} \in \mathcal{L}(X, Y) \times Y$ and $k \in \operatorname{int} K$. It follows from Proposition 5.3 that the qualifying conditions $\left(\mathrm{a}_{1}\right)$ and $\left(\mathrm{b}_{1}\right)$ in Theorem 5.3, and $\left(\mathrm{c}_{k}\right)$ in Theorem 5.5 hold. The conclusion follows from Theorems 5.3, 5.5.

\section{Duality for robust convex vector optimization problems}

In this section, concerning the robust vector optimization problem (RVP) defined by (4.1) with its feasible set $A$ as in (4.2) with the assumption that $A \cap \operatorname{dom} F \neq \emptyset$,we define a new kind of Lagrange dual problems $\left(\mathrm{RVD}^{k}\right)$ based on some results $k$-sectional convexity with $k \in \operatorname{int} K$, along with the Lagrangian robust dual problem (RVD) and the weak Lagrangian robust dual problem $\left(\mathrm{RVD}_{w}\right)$ introduced in [10]. We will establish several robust strong stable duality results for the pairs (RVP)-(RVD), (RVP)-(RVD ${ }_{w}$, 
and (RVP)-(RVD $\left.{ }^{k}\right)$. The results on robust strong stable duality for the pair (RVP)$\left(\mathrm{RVD}^{k}\right)$ are new while the ones for other dual pairs are established under qualification conditions which are different from [10] and some how are easier to check than the ones in [10].

Recall that $\mathcal{A}, \mathcal{B}$ and $\mathcal{A}_{k}$ (for some $k \in \operatorname{int} K$ ) the qualifying sets defined respectively by (4.3), (4.4), and (4.5).

\subsection{Lagrange duality for robust vector optimization problems}

We consider the Lagrangian robust dual problem (RVD) and the weak Lagrangian robust dual problem $\left(\mathrm{RVD}_{w}\right)$ [10] of (RVP) defined respectively by

$$
\begin{array}{ll}
(\mathrm{RVD}) & \operatorname{WSup}_{(T, u) \in \mathcal{L}_{+}(S, K) \times \mathcal{U}} \operatorname{WInf}_{x \in C}\left(F+T \circ G_{u}\right)(x), \\
\left(\mathrm{RVD}_{w}\right) & \operatorname{WSup}_{(T, u) \in \mathcal{L}_{+}^{w}(S, K) \times \mathcal{U}} \operatorname{WInf}_{(x, s) \in C \times S}\left[\left(F+T \circ G_{u}\right)(x)+T(s)\right] .
\end{array}
$$

We say that the robust strong duality holds for the pair (RVP) - (RVD) (resp., for the pair (RVP) - (RVDw)) if the sets of values of the two problems (RVP) and (RVD) (resp., of the two problems $(\mathrm{RVP})$ and $\left(\mathrm{RVD}_{w}\right)$ ) are equal together, that is,

$$
\operatorname{WMax}(\mathrm{RVD})=\mathrm{WInf}(\mathrm{RVP}) \quad\left(\text { resp., } \operatorname{WMax}\left(\mathrm{RVD}_{w}\right)=\operatorname{WInf}(\mathrm{RVP})\right) .
$$

For $L \in \mathcal{L}(X, Y)$, we denote by $\left(\mathrm{RVP}^{L}\right)$ the perturbed vector problem

$$
\left(\mathrm{RVP}^{L}\right) \quad \text { WMin }\left\{F(x)-L(x): x \in C, G_{u}(x) \in-S, \forall u \in \mathcal{U}\right\} .
$$

Then, the Lagrangian robust dual problem and the weak Lagrangian robust dual problem of $\left(\mathrm{RVP}^{L}\right)$ are, respectively,

$$
\begin{array}{ll}
\left(\operatorname{RVD}^{L}\right) & \operatorname{WSup}_{(T, u) \in \mathcal{L}_{+}(S, K) \times \mathcal{U}} \operatorname{Winf}_{x \in C}\left(F-L+T \circ G_{u}\right)(x), \\
\left(\operatorname{RVD}_{w}^{L}\right) & \operatorname{WSup}_{(T, u) \in \mathcal{L}_{+}^{w}(S, K) \times \mathcal{U}} \operatorname{WInf}_{(x, s) \in C \times S}\left[\left(F-L+T \circ G_{u}\right)(x)+T(s)\right] .
\end{array}
$$

Let $\emptyset \neq \mathcal{V} \subset \mathcal{L}(X, Y)$. We say that the robust strong $\mathcal{V}$-stable duality holds for the pair (RVP) - (RVD) if, for any $L \in \mathcal{V}$,

$$
\operatorname{WMax}\left(\operatorname{RVD}^{L}\right)=\mathrm{WInf}\left(\mathrm{RVP}^{L}\right) \text {. }
$$

When $\mathcal{V}=\mathcal{L}(X, Y)$ we will say that the robust strong stable duality holds for the pair (RVP) - (RVD) instead of "the robust strong $\mathcal{L}(X, Y)$-stable duality holds for the pair (RVP) - (RVD)". It is obviously that when $\mathcal{V}=\left\{0_{\mathcal{L}}\right\}$, the concept "robust strong $\mathcal{V}$-stable duality" reduces to the concept "robust strong duality". For the pair $(\mathrm{RVP})-\left(\mathrm{RVD}_{w}\right)$, the corresponding concepts (for instance, robust strong $\mathcal{V}$-stable duality holds for the pair (RVP) - $\left.\left(\mathrm{RVD}_{w}\right)\right)$ will be defined in the same way.

We first introduce the following principles of robust strong $\mathcal{V}$-stable duality.

Theorem 6.1 (Principles of robust strong $\mathcal{V}$-stable duality I). Consider the following statements:

(e) $\operatorname{epi}\left(F+I_{A}\right)^{*} \cap(\mathcal{V} \times Y)=\mathcal{A} \cap(\mathcal{V} \times Y)$,

(f) $\operatorname{epi}\left(F+I_{A}\right)^{*} \cap(\mathcal{V} \times Y)=\mathcal{B} \cap(\mathcal{V} \times Y)$,

(g) The robust strong $\mathcal{V}$-stable duality holds for the pair (RVP) - (RVD), 
(h) The robust strong $\mathcal{V}$-stable duality holds for the pair $(\mathrm{RVP})-\left(\mathrm{RVD}_{w}\right)$. Then, one has $[(\mathrm{e}) \Longleftrightarrow(\mathrm{g})]$ and $[(\mathrm{f}) \Longleftrightarrow(\mathrm{h})]$.

Proof. Proof of $[(\mathrm{e}) \Longrightarrow(\mathrm{g})]$. Take $L \in \mathcal{V}$, we will prove that $\operatorname{WInf}\left(\mathrm{RVP}^{L}\right)=$ WMax $\left(\operatorname{RVD}^{L}\right)$. Firstly, it is worth noting that the problems $\left(\operatorname{RVP}^{L}\right)$ and $\left(\operatorname{RVD}^{L}\right)$ are respectively nothing else but the problems (RVP) and (RVD) with $F$ replaced by $F-L$. As (e) holds, according to Theorem 5.1, it holds, for all $y \in Y$,

$$
\begin{gathered}
\left(G_{u}(x) \in-S, x \in C, \forall u \in \mathcal{U} \Longrightarrow y-L(x)+F(x) \notin-\operatorname{int} K\right) \\
\mathbb{\mathbb { }} \Longrightarrow \\
\left(\exists u \in \mathcal{U}, \exists T \in \mathcal{L}_{+}(S, K): F(x)+T \circ G_{u}(x)-L(x)+y \notin-\operatorname{int} K, \forall x \in C\right),
\end{gathered}
$$

or in other words, [10, Theorem $1(i i)]$ holds with $F-L$ replacing $F$. Repeat whole the proof of $[(\mathrm{a}) \Longrightarrow(\mathrm{b})]$ of [10, Theorem 6], we obtain $\operatorname{WInf}\left(\operatorname{RVP}^{L}\right)=\operatorname{WMax}\left(\operatorname{RVD}^{L}\right)$.

Proof of $[(\mathrm{e}) \Longleftarrow(\mathrm{g})]$. It follows from Proposition 4.1 that

$$
\mathcal{A} \cap(\mathcal{V} \times \mathcal{W}) \subset \operatorname{epi}\left(F+I_{A}\right)^{*} \cap(\mathcal{V} \times \mathcal{W})
$$

So, to prove (e) holds, it is sufficient to check that the converse inclusion of (6.4) holds. Take $(L, y) \in \operatorname{epi}\left(F+I_{A}\right)^{*} \cap(\mathcal{V} \times \mathcal{W})$. Then, according to (2.9),

$$
y+F(x)-L(x) \notin-\operatorname{int} K, \forall x \in A \cap \operatorname{dom} F .
$$

Use the same argument as in the proof of (51) of [10] (page 312) with $F-L$ replacing $F$, one gets the existence of $(T, u) \in \mathcal{L}_{+}(S, K) \times \mathcal{U}$ such that $(L, y) \in \operatorname{epi}\left(F+I_{C}+T \circ G_{u}\right)^{*} \subset$ $\mathcal{A}$. So, the converse inclusion of (6.4) holds.

Proof of $[(\mathrm{f}) \Longleftrightarrow(\mathrm{h})]$. The proof is similar to the one of $[(\mathrm{e}) \Longleftrightarrow(\mathrm{g})]$.

We now turn to the convex case i.e., the case where $\left(\mathcal{H}_{0}\right)$ holds. In such a case, with the help of the results established in Section 4, the qualifying conditions in (e) and (f) can be described in terms of sectional convexity and closedness.

Theorem 6.2 (Principles of convex robust strong $\mathcal{V}$-stable duality I). Assume that $\left(\mathcal{H}_{0}\right)$ holds. Consider the following statements:

$\left(\mathrm{e}_{1}\right) \exists k \in \operatorname{int} K$ s.t. $\mathcal{A}$ is $k$-sectionally convex and closed regarding $\mathcal{V} \times Y$,

$\left(\mathrm{f}_{1}\right) \exists k \in \operatorname{int} K$ s.t. $\mathcal{B}$ is $k$-sectionally convex and closed regarding $\mathcal{V} \times Y$.

Then, one has $\left[\left(\mathrm{e}_{1}\right) \Longleftrightarrow(\mathrm{g})\right]$ and $\left[\left(\mathrm{f}_{1}\right) \Longleftrightarrow(\mathrm{h})\right]$, where $(\mathrm{g})$ and $(\mathrm{h})$ are in Theorem 6.1 .

Proof. As $\left(\mathcal{H}_{0}\right)$ holds, it follows from Theorem 4.2 that

$$
\operatorname{epi}\left(F+I_{A}\right)^{*}=\operatorname{cl}\left(\operatorname{sco}_{k} \mathcal{A}\right)=\operatorname{cl}\left(\operatorname{sco}_{k} \mathcal{B}\right), \forall k \in \operatorname{int} K
$$

So, the statements $\left(\mathrm{e}_{1}\right)$ and $\left(\mathrm{f}_{1}\right)$ are equivalent to epi $\left(F+I_{A}\right)^{*} \cap(\mathcal{V} \times Y)=\mathcal{A} \cap(\mathcal{V} \times Y)$ and epi $\left(F+I_{A}\right)^{*} \cap(\mathcal{V} \times Y)=\mathcal{B} \cap(\mathcal{V} \times Y)$, respectively. The conclusion now follows from Theorem 6.1 .

Theorem 6.3 (Convex robust strong $\mathcal{V}$-stable duality). Assume that $\left(\mathcal{H}_{0}\right)$ and the following condition hold:

$\left(\mathrm{e}_{2}\right) \exists k \in \operatorname{int} K$ s.t. $\mathcal{A}_{k}$ is $k$-sectionally convex and closed regarding $\mathcal{V} \times Y$. 
Then, strong robust $\mathcal{V}$-stable duality holds for $(\mathrm{RVP})-(\mathrm{RVD})$ and $(\mathrm{RVP})-\left(\mathrm{RVD}_{w}\right)$.

Proof. Use the same argument as in the proof of Theorem 5.4 (with $\mathcal{W}=Y$ ) we can show that if $\left(\mathrm{e}_{2}\right)$ holds then (e) and (f) in Theorem 6.1 hold and then, the conclusion follows from Theorem 6.1.

Theorem 6.4 (Convex robust strong stable duality I). Assume that $\mathcal{U}$ is a compact space, that $Z$ is a normed space, and that the hypotheses $\left(\mathcal{H}_{0}\right),\left(\mathcal{H}_{1}\right),\left(\mathcal{H}_{2}\right)$, and the Slater-type condition $\left(C_{0}\right)$ hold. Then robust strong stable duality holds for two pairs $(\mathrm{RVP})-(\mathrm{RVD})$ and $(\mathrm{RVP})-\left(\mathrm{RVD}_{w}\right)$.

Proof. It follows from Proposition 5.3 and Theorem 6.2.

Remark 6.1. It worth observing that the hypotheses $\left(\mathcal{H}_{1}\right),\left(\mathcal{H}_{2}\right)$ and the condition $\left(C_{0}\right)$ do not concern the objective mapping $F$. So, the conclusion of Theorem 6.4 until holds true when $F$ is replaced by arbitrary proper $K$-convex and positively $K$-lsc. In other words, under the assumptions of Theorem 6.4, the robust strong duality for pairs $(\mathrm{RVP})-(\mathrm{RVD})$ and $(\mathrm{RVP})-\left(\mathrm{RVD}_{w}\right)$ are stable in a stronger sense that the objective mapping $F$ can be perturbed by arbitrary mapping provided that properties: "proper", " $K$-convex", and "positively $K$-lsc" are still reserved.

\subsection{Robust duality via $k$-sectional convexity}

Fix $k \in \operatorname{int} K$. By letting $\mathcal{A}_{k}$ play the role of $\mathcal{A}$ (or $\mathcal{B}$ ) as the qualifying set, one gets the dual problem $\left(\mathrm{RVD}^{k}\right)$ as follows:

$$
\left(\operatorname{RVD}^{k}\right) \quad \operatorname{WSup}_{\left(z^{*}, u\right) \in S^{+} \times \mathcal{U}} \underset{x \in C}{\operatorname{Winf}}\left[F(x)+\left(z^{*} \circ G_{u}\right)(x) k\right] .
$$

The robust strong duality and the $\mathcal{V}$-stable robust strong duality for pair (RVP) $-\left(\mathrm{RVD}^{k}\right)$ can be understood by the same way as the previous subsection.

Theorem 6.5 (Principles of robust strong $\mathcal{V}$-stable duality II). Let $\emptyset \neq \mathcal{V} \subset Y$. The following statements are equivalent:

$\left(\mathrm{e}_{k}\right) \operatorname{epi}\left(F+I_{A}\right)^{*} \cap(\mathcal{V} \times Y)=\mathcal{A}_{k} \cap(\mathcal{V} \times Y)$,

$\left(\mathrm{g}_{k}\right)$ The robust strong $\mathcal{V}$-stable duality holds for the pair $(\mathrm{RVP})-\left(\mathrm{RVD}^{k}\right)$.

Proof. Use the same argument as in proof of $[(\mathrm{e}) \Longleftrightarrow(\mathrm{g})]$ in Theorem 6.1, using Theorem 5.2 instead of Theorem 5.1 .

Theorem 6.6 (Principle of convex robust strong $\mathcal{V}$-stable duality II). Let $\emptyset \neq \mathcal{V} \subset Y$. Assume $\left(\mathcal{H}_{0}\right)$ hold and consider the following statement:

$\left(\mathrm{e}_{k}^{\prime}\right) \mathcal{A}_{k}$ is $k$-sectionally convex and closed regarding $\mathcal{V} \times Y$.

Then, $\left[\left(\mathrm{e}_{k}^{\prime}\right) \Longleftrightarrow\left(\mathrm{g}_{k}\right)\right]$, where $\left(\mathrm{g}_{k}\right)$ is the statement in Theorem 6.5.

Proof. As $\left(\mathcal{H}_{0}\right)$ is satisfied, Theorem 4.1 gives epi $\left(F+I_{A}\right)^{*}=\operatorname{cl}\left(\operatorname{sco}_{k} \mathcal{A}_{k}\right)$ and so, $\left(\mathrm{e}_{k}^{\prime}\right)$ in this case is nothing else but $\left(\mathrm{e}_{k}\right)$ in Theorem 6.5. The conclusion now follows from Theorem 6.5. 
Theorem 6.7 (Convex robust strong stable duality II). Assume that $\mathcal{U}$ is a compact space, that $Z$ is a normed space, and that the hypotheses $\left(\mathcal{H}_{0}\right),\left(\mathcal{H}_{1}\right),\left(\mathcal{H}_{2}\right)$ and the Slater-type condition $\left(C_{0}\right)$ hold. Then strong robust stable duality holds for pair (RVP)$\left(\mathrm{RVD}^{k}\right)$.

Proof. It follows from Proposition 5.3 and Theorem 6.6.

\section{Applications to robust convex optimizations}

In this section we will specialize our results on robust strong (stable) duality for vector problems obtained in Section 6 to some classes of (scalar) robust convex optimizations, which means that we will consider the case when $Y=\mathbb{R}$ and $K=\mathbb{R}_{+}$(and hence, $\mathbb{R}^{\bullet} \equiv \overline{\mathbb{R}}:=\mathbb{R} \cup\{ \pm \infty\}$ ). In this setting, we will write $f$ (instead of $F$ ) for the objective function of problems. Observed also that in this case $\mathcal{L}(X, Y)$ becomes $X^{*}$, both the cones $\mathcal{L}_{+}(S, K)$ and $\mathcal{L}_{+}^{w}(S, K)$ now collapse to the positive dual cone $S^{+}$of $S$, and the conjugate $f^{*}$ is none other than the usual conjugate $f^{*}$ in the sense of convex analysis. As results, the specification even to robust scalar problems still produce some new robust strong duality results, some that extend, or cover the known ones in the literature.

\subsection{General robust convex optimization problem}

Consider the robust convex optimization problem:

$(\mathrm{RP}) \quad \inf \left\{f(x): x \in C, G_{u}(x) \in-S, \forall u \in \mathcal{U}\right\}$

where $X, Z$ are lcHtvs, $S$ is a closed convex cone of $Z, \mathcal{U}$ is an uncertainty set, $f \in \Gamma(X)$, $G_{u}: X \rightarrow Z^{\bullet}$ is proper, $S$-convex and $S$-epi closed mapping for all $u \in \mathcal{U}$, and $C \subset X$ is a nonempty closed and convex subset of $X$. Note that under these assumptions, $\left(\mathcal{H}_{0}\right)$ is satisfied.

Let us retain call $A$ (in (4.2) ) the feasible set of (RP). Assume that $A \cap \operatorname{dom} f \neq \emptyset$.

For the problem (RP), the qualifying sets $\mathcal{A}, \mathcal{B}$, and $\mathcal{A}_{k}$ (for any $k \in \operatorname{int} \mathbb{R}_{+}$) in Section 4 collapse to the unique one

$$
\widehat{\mathcal{A}}:=\bigcup_{\left(z^{*}, u\right) \in S^{+} \times \mathcal{U}} \operatorname{epi}\left(f+i_{C}+z^{*} \circ G_{u}\right)^{*} .
$$

The Lagrangian dual problem (RVD), the weak Lagrangian dual problem $\left(\mathrm{RVD}_{w}\right)$ and dual problem $\left(\mathrm{RVD}^{k}\right)$ in this case collapse to the unique Lagrange dual problem (RD) of (RP):

$$
\sup _{\left(z^{*}, u\right) \in S^{+} \times \mathcal{U}} \inf _{x \in C}\left(f+z^{*} \circ G_{u}\right)(x)
$$

and, for all $\emptyset \neq \mathcal{V} \subset X^{*}$, "the robust strong $\mathcal{V}$-stable duality holds for the pair $(\mathrm{RP})-(\mathrm{RD})$ " means that, for all $x^{*} \in \mathcal{V}$, $\inf \left\{f(x)-\left\langle x^{*}, x\right\rangle: x \in C, G_{u}(x) \in-S, \forall u \in \mathcal{U}\right\}=\max _{\left(z^{*}, u\right) \in S^{+} \times \mathcal{U}} \inf _{x \in C}\left(f-x^{*}+z^{*} \circ G_{u}\right)(x)$. The next two corollaries come directly from Theorem 6.2 and Theorem 6.4, respectively. 
Corollary 7.1. [12, Theorem 6.3](Principle of robust convex strong $\mathcal{V}$-stable duality) Let $\emptyset \neq \mathcal{V} \subset X^{*}$. The following statements are equivalent:

(k) The set $\widehat{\mathcal{A}}$ is closed and convex regarding $\mathcal{V} \times \mathbb{R}$,

(l) The robust strong $\mathcal{V}$-stable duality holds for the pair $(\mathrm{RP})-(\mathrm{RD})$.

Corollary 7.2. Assume that $\mathcal{U}$ is a compact space, that $Z$ is a normed space, and that the hypotheses $\left(\mathcal{H}_{1}\right)$ and $\left(\mathcal{H}_{2}\right)$ in Section 5 hold. Assume further that the following condition holds:

$\left(\widehat{\mathrm{C}}_{0}\right) \forall u \in \mathcal{U}, \exists x_{u} \in C \cap \operatorname{dom} f: G_{u}\left(x_{u}\right) \in-\operatorname{int} S$.

Then, the robust strong $\mathcal{V}$-stable duality holds for the pair $(\mathrm{RP})-(\mathrm{RD})$.

\subsection{Robust convex programming under uncertain inequality constraints}

Consider the robust convex programming of the form

$$
(\mathrm{RCP}) \quad \inf \left\{f(x): x \in C, g_{t}\left(x, u_{t}\right) \leq 0, \forall u_{t} \in U_{t}, \forall t \in T\right\}
$$

where $f \in \Gamma(X), T$ is a possibly infinite index set, $U_{t}$ is uncertainty set for each $t \in T$, $g_{t}\left(., u_{t}\right) \in \Gamma(X)$ for all $u_{t} \in U_{t}$ and $t \in T$, and $C \subset X$ is nonempty closed and convex. Let $A:=\left\{x \in C: g_{t}\left(x, u_{t}\right) \leq 0, \forall u_{t} \in U_{t}, \forall t \in T\right\}$ and assume that $A \cap \operatorname{dom} f \neq \emptyset$.

We will propose several ways to transform (RCP) to the form of (RP). The robust strong (stable) duality results in the previous subsection are then applied to get the variants of robust strong duality results for (RCP), which are new, extend or cover the known ones in the literature.

- The first way: Take $Z=\mathbb{R}^{T}, S=\mathbb{R}_{+}^{T}, \mathcal{U}=\prod_{t \in T} U_{t}, G_{u}(x)=\left(g_{t}\left(x, u_{t}\right)\right)_{t \in T}$ for all $x \in X$ and $u=\left(u_{t}\right)_{t \in T} \in \mathcal{U}$. We consider $\mathbb{R}^{T}$ endowed with the product topology and its dual space, $\mathbb{R}^{(T)}$, is the space of generalized finite sequences (i.e., the functions $\lambda=\left(\lambda_{t}\right)_{t \in T} \in \mathbb{R}^{T}$ such that its supporting set $\operatorname{supp} \lambda:=\left\{t \in T: \lambda_{t} \neq 0\right\}$ is finite) with dual product defined by

$$
\langle\lambda, v\rangle:= \begin{cases}\sum_{t \in \operatorname{supp} \lambda} \lambda_{t} v_{t}, & \text { if } \lambda \neq 0_{T}, \\ 0, & \text { otherwise }\end{cases}
$$

for all $(\lambda, v) \in \mathbb{R}^{(T)} \times \mathbb{R}^{T}$. The positive cones in $\mathbb{R}^{T}$ and in $\mathbb{R}^{(T)}$ is denoted by $\mathbb{R}_{+}^{T}$ and $\mathbb{R}_{+}^{(T)}$, respectively. In this setting, the qualifying set $\widehat{\mathcal{A}}$ becomes

$$
\widehat{\mathcal{A}}_{1}:=\bigcup_{\substack{\left(\lambda_{t}\right)_{t \in T} \in \mathbb{R}_{+}^{(T)} \\\left(u_{t}\right)_{t \in T} \times \mathcal{U}}} \operatorname{epi}\left(f+i_{C}+\sum_{t \in T} \lambda_{t} g_{t}\left(., u_{t}\right)\right)^{*}
$$

and the robust dual problem $(\mathrm{RD})$ now becomes

$$
\left(\mathrm{RCD}_{1}\right) \quad \sup _{\substack{\left(\lambda_{t}\right)_{t \in T} \in \mathbb{R}_{+}^{(T)} \\\left(u_{t}\right)_{t \in T} \times \mathcal{U}}} \inf _{x \in C}\left(f(x)+\sum_{t \in T} \lambda_{t} g_{t}\left(x, u_{t}\right)\right) .
$$

The robust dual problem of this form was considered in other works as [10, 13, 16, 17]. The next corollary is a direct consequence of Corollary 7.1 which turns back to [12, Theorem 6.4] and covers [13, Theorem 4.1] (for $i=O$ ) and [16, Theorem 3.1]. 
Corollary 7.3. Let $\emptyset \neq \mathcal{V} \subset X^{*}$. The following statements are equivalent:

$\left(\mathrm{k}_{1}\right)$ The set $\widehat{\mathcal{A}}_{1}$ is closed and convex regarding $\mathcal{V} \times \mathbb{R}$,

$\left(\mathrm{l}_{1}\right)$ The robust strong $\mathcal{V}$-stable duality holds for $(\mathrm{RCP})-\left(\mathrm{RCD}_{1}\right)$, i.e., for all $x^{*} \in \mathcal{V}$,

$$
\inf _{\substack{x \in C \\ g_{t}\left(x, u_{t}\right) \leq 0, \forall u_{t} \in U_{t}, \forall t \in T}}\left[f(x)-\left\langle x^{*}, x\right\rangle\right]=\max _{\substack{\left(\lambda_{t}\right)_{t \in T} \in \mathbb{R}_{+}^{(T)} \\\left(u_{t}\right)_{t \in T} \times \mathcal{U}}} \inf _{x \in C}\left(f(x)-\left\langle x^{*}, x\right\rangle+\sum_{t \in T} \lambda_{t} g_{t}\left(x, u_{t}\right)\right) .
$$

The next result is a consequence of Corollary 7.2 and extends [16, Corollary 3.3].

Corollary 7.4. Assume that $T$ is finite and that $U_{t}$ is a compact and convex subset of some topological vector space for all $t \in T$, and $g_{t}(x,.) \in-\Gamma\left(U_{t}\right)$ for all $x \in C \cap \operatorname{dom} f$. Assume further that the following condition holds:

$\left(\widehat{\mathrm{C}}_{0}^{1}\right) \forall u=\left(u_{t}\right)_{t \in T} \in \mathcal{U}, \exists x_{u} \in C \cap \operatorname{dom} f: g_{t}\left(x_{u}, u_{t}\right)<0, \forall t \in T$.

Then, the robust strong stable duality holds for the pair $(\mathrm{RCP})-\left(\mathrm{RCD}_{1}\right)$.

Proof. Firstly, $\mathcal{U}$ is compact (as $U_{t}$ is compact for all $t \in T$ ) and $Z:=\mathbb{R}^{T}$ is a normed space (note that $T$ is finite).

- We now prove that the collection $\left(\left(u_{t}\right)_{t \in T}^{m} \mapsto\left(g_{t}\left(x, u_{t}\right)\right)_{t \in T}\right)_{x \in C \cap \operatorname{dom} f}$ is uniformly $\mathbb{R}_{+}^{(T)}$-concave, or equivalently, the hypothesis $\left(\mathcal{H}_{1}\right)$ holds. For this, take $\left(\lambda_{t}^{j}\right)_{t \in T} \in \mathbb{R}_{+}^{(T)}$ and $\left(u_{t}^{j}\right)_{t \in T} \in \mathcal{U}(j=1,2)$, we will find $\left(\bar{\lambda}_{t}\right)_{t \in T} \in \mathbb{R}_{+}^{(T)}$ and $\left(\bar{u}_{t}\right)_{t \in T} \in \mathcal{U}$ such that

$$
\sum_{t \in T} \lambda_{t}^{1} g_{t}\left(x, u_{t}^{1}\right)+\sum_{t \in T} \lambda_{t}^{2} g_{t}\left(x, u_{t}^{2}\right) \leq \sum_{t \in T} \bar{\lambda}_{t} g_{t}\left(x, \bar{u}_{t}\right), \quad \forall x \in C \cap \operatorname{dom} f .
$$

To do this, for all $t \in T$, take $\bar{\lambda}_{t}:=\lambda_{t}^{1}+\lambda_{t}^{2}$ and

$$
\bar{u}_{t}:= \begin{cases}\frac{\lambda_{t}^{1}}{\lambda_{t}^{1}+\lambda_{i}^{2}} u_{t}^{1}+\frac{\lambda_{t}^{2}}{\lambda_{t}^{1}+\lambda_{t}^{2}} u_{t}^{2}, & \text { if } \lambda_{t}^{1}>0 \text { or } \lambda_{t}^{2}>0 \\ u_{t}^{1} & \text { else. }\end{cases}
$$

As $g_{t}(x,$.$) is concave on the convex set U_{t}$ for each $t \in T$, one has (see Example 3.1), for all $x \in C \cap \operatorname{dom} f$,

$$
\lambda_{t}^{1} g_{t}\left(x, u_{t}^{1}\right)+\lambda_{t}^{2} g_{t}\left(x, u_{t}^{2}\right) \leq \bar{\lambda}_{t} g_{t}\left(x, \bar{u}_{t}\right)
$$

which, in turn, yields (7.2).

- Next, for all $x \in C \cap \operatorname{dom} f$, as $g_{t}(x,):. U_{t} \subset \mathbb{R}^{q_{i}} \rightarrow \mathbb{R}$ is usc for all $t \in T$, by Lemma 3.1(iii)), $\left(g_{t}(x, .)\right)_{t \in T}$ is $\mathbb{R}_{+}^{(T)}$-uniformly usc, meaning that $\left(\mathcal{H}_{2}\right)$ holds.

- Finally, the fulfilment of $\left(\widehat{C}_{0}^{1}\right)$ entails that the Slater-type condition $\left(\widehat{C}_{0}\right)$ in Corollary 7.2 holds. The conclusion now follows from Corollary 7.2 .

- The second way: Take $Z=\mathbb{R}, \mathcal{U}=T$, and $G_{t}(x)=\sup _{v \in U_{t}} g_{t}(x, v)$ for all $x \in X$ and $t \in T$. Then, the qualifying set $\widehat{\mathcal{A}}$ becomes

$$
\widehat{\mathcal{A}}_{2}=\bigcup_{\lambda \geq 0, t \in T} \operatorname{clco}\left(\bigcup_{v \in U_{t}} \operatorname{epi}\left(f+i_{C}+\lambda g_{t}(., v)\right)^{*}\right)
$$

(see [18, Lemma 2.2]). The robust dual problem (RD) now reduces to

$$
\left(\mathrm{RCD}_{2}\right) \quad \sup _{\lambda \geq 0, t \in T} \inf _{x \in C} \sup _{v \in U_{t}}\left(f(x)+\lambda g_{t}(x, v)\right) .
$$


This form of robust dual problem of (RCIP) is proposed in [13, Remark 10]. As consequences of Corollaries 7.1/7.2, one gets.

Corollary 7.5. Let $\emptyset \neq \mathcal{V} \subset X^{*}$. The following statements are equivalent:

$\left(\mathrm{k}_{2}\right)$ The set $\widehat{\mathcal{A}}_{2}$ is closed and convex regarding $\mathcal{V} \times \mathbb{R}$,

$\left(\mathrm{l}_{2}\right)$ The robust strong $\mathcal{V}$-stable duality holds for the pair $(\mathrm{RCP})-\left(\mathrm{RCD}_{2}\right)$.

Corollary 7.6. Assume that $T$ is a compact and convex subset of some topological vector space, and that the function $t \mapsto \sup _{v \in U_{t}} g_{t}(x, v)$ is concave and usc on $T$ for all $x \in C \cap \operatorname{dom} f$. Assume further that the following condition holds:

$\left(\widehat{\mathrm{C}}_{0}^{2}\right) \forall t \in T, \exists x_{t} \in C \cap \operatorname{dom} f: \sup _{v \in U_{t}} g_{t}\left(x_{u}, v\right)<0$.

Then, the robust strong stable duality holds for the pair $(\mathrm{RCP})-\left(\mathrm{RCD}_{2}\right)$.

- The third way: Take $Z=\mathbb{R}, \mathcal{U}=\prod_{t \in T} U_{t}$, and $G_{u}(x)=\sup _{t \in T} g_{t}\left(x, u_{t}\right)$ for all $x \in X$ and $u=\left(u_{t}\right)_{t \in T}$. Then, the qualifying set $\widehat{\mathcal{A}}$ becomes

$$
\widehat{\mathcal{A}}_{3}=\bigcup_{\substack{\lambda \geq 0,\left(u_{t}\right)}} \operatorname{clco}\left(\bigcup_{t \in T} \operatorname{epi}\left(f+i_{C}+\lambda g_{t}\left(., u_{t}\right)\right)^{*}\right)
$$

and the robust dual problem (RD) of of (RCP) turns to new form as follows:

$$
\left(\mathrm{RCD}_{3}\right) \quad \sup _{\substack{\lambda \geq 0,\left(u_{t}\right)}} \inf _{x \in T} \sup _{t \in T}\left(f(x)+\lambda g_{t}\left(x, u_{t}\right)\right) .
$$

Now, Corollaries $7.1+7.2$ gives us the next results.

Corollary 7.7. Let $\emptyset \neq \mathcal{V} \subset X^{*}$. The following statements are equivalent:

$\left(\mathrm{k}_{3}\right)$ The set $\widehat{\mathcal{A}}_{3}$ is closed and convex regarding $\mathcal{V} \times \mathbb{R}$,

$\left(\mathrm{l}_{3}\right)$ The robust strong $\mathcal{V}$-stable duality holds for the pair $(\mathrm{RCP})-\left(\mathrm{RCD}_{3}\right)$.

Corollary 7.8. Assume that $U_{t}$ is a compact and convex subset of some vector topological space for all $t \in T$, and that the function $\left(u_{t}\right)_{t \in T} \mapsto \sup _{t \in T} g_{t}\left(x, u_{t}\right)$ is concave and usc on $\mathcal{U}$ for all $x \in C \cap \operatorname{dom} f$. Assume further that the following condition holds: $\left(\widehat{\mathrm{C}}_{0}^{3}\right) \forall u=\left(u_{t}\right)_{t \in T} \in \mathcal{U}, \exists x_{u} \in C \cap \operatorname{dom} f: \sup _{t \in T} g_{t}\left(x_{u}, u_{t}\right)<0$.

Then, the robust strong stable duality holds for the pair $(\mathrm{RCP})-\left(\mathrm{RCD}_{3}\right)$.

Remark 7.1. Noting that there are still other ways of transforming (RCP) to the form of $(\mathrm{RP})$. For instance, take $\mathfrak{U}=\left\{\left(t, u_{t}\right): t \in T, u_{t} \in U_{t}\right\}, Z=\mathbb{R}^{\mathfrak{U}}, S=\mathbb{R}_{+}^{\mathfrak{U}}, \mathcal{U}=\{\mathfrak{U}\}$ and $G_{\mathfrak{U}}=\left(g_{t}\left(., u_{t}\right)\right)_{\left(t, u_{t}\right) \in \mathfrak{U}}$. Then, $\widehat{\mathcal{A}}$ and the dual problem $(\mathrm{RD})$ become, respectively

$$
\begin{gathered}
\widehat{\mathcal{A}}_{4}=\bigcup_{\lambda \in \mathbb{R}_{+}^{(\mathfrak{H})}} \operatorname{epi}\left(f+i_{C}+\sum_{\left(t, u_{t}\right) \in \mathfrak{U}} \lambda_{\left(t, u_{t}\right)} g_{t}\left(., u_{t}\right)\right)^{*}, \\
\left(\mathrm{RCD}_{4}\right) \sup _{\lambda \in \mathbb{R}_{+}^{(\mathfrak{L})}} \inf _{x \in C}\left(f(x)+\sum_{\left(t, u_{t}\right) \in \mathfrak{U}} \lambda_{\left(t, u_{t}\right)} g_{t}\left(x, u_{t}\right)\right)^{*} .
\end{gathered}
$$

By Proposition 3.4, $\widehat{\mathcal{A}}_{4}$ is a convex subset of $X^{*} \times \mathbb{R}$. So, Corollary 7.1 yields the equivalence of two following assertions: 
$\left(\mathrm{k}_{4}\right)$ The set $\widehat{\mathcal{A}}_{4}$ is closed regarding $\mathcal{V} \times \mathbb{R}$,

$\left(l_{4}\right)$ The robust strong $\mathcal{V}$-stable duality holds for the pair $(\mathrm{RCP})-\left(\mathrm{RCD}_{4}\right)$.

This result covers [13, Theorem 4.1] for the case $i=C$. By using other suitable ways, we can get results that possibly cover [13, Theorem 4.1] with other values of $i$.

\section{References}

[1] Aliprantis, ChD, Burkinshaw, O.: Positive Operators. Academic Press, Orlando (1985)

[2] Barro, M., Ouédraogo, A., Traoré, S.: On Uncertain Conical Convex Optimization Problems. Pacific J. Optim. 13, 29-42 (2017)

[3] Beck, A., Ben-Tal, A.: Duality in robust optimization: Primal worst equals dual best. Oper. Res. Lett. 37, 1-6 (2009)

[4] Ben-Tal, A., El Ghaoui, L., Nemirovski, A.: Robust Optimization. Princeton U.P., Princeton (2009)

[5] Bertsimas, D., Brown, D.B., Caramanis, C.: Theory and applications of robust optimization. SIAM Rev. 53, 464-501 (2011)

[6] Bot, R.I.: Conjugate Duality in Convex Optimization. Springer, Berlin (2010)

[7] Bot, R.I., Grad, S.M., Wanka, G.: Duality in Vector Optimization. SpringerVerlag, Berlin, Germany (2009)

[8] Dinh, N., Goberna, M.A., López, M.A., Mo, T.H.: Farkas-type results for vectorvalued functions with applications. J. Optim. Theory Appl. 173, 357-390 (2017)

[9] Dinh, N., Goberna, M.A., López, M.A., Mo, T.H.: Robust optimization revisited via robust vector Farkas lemmas. Optimization 66, 939-963 (2017)

[10] Dinh, N., Long, D.H.: Complete characterizations of robust strong duality for robust vector optimization problems. Vietnam J. Math. 46, 293-328 (2018)

[11] Dinh, N., Goberna, M.A., Long, D.H., López, M.A.: New Farkas-type results for vector-valued functions: a non-abstract approach. J. Optim. Theory Appl. (to appear). https://doi.org/10.1007/s10957-018-1352-z

[12] Dinh, N., Mo, T.H., Vallet, G., Volle, M.: A unified approach to robust Farkastype results with applications to robust optimization problems. SIAM J. Optim. 27, 1075-1101 (2017)

[13] Dinh, N., Goberna, M.A., Lopez, M.A., Volle, M.: A unifying approach to robust convex infinite optimization duality. J. Optim. Theory Appl. 174, 650 - 685 (2017) 
[14] Goberna, M. A., Jeyakumar, V., Li, G., Lopez, M. A: Robust linear semi-infinite programming duality under uncertainty. Math. Program. 139, Ser. B, 185-203 (2013)

[15] Gabrel, V., Murat, C., Thiele, A.: Recent advances in robust optimization: An overview. European J. Oper Res., 235, 471-483 (2014)

[16] Jeyakumar, V., Li, G.Y.: Strong duality in robust convex programming: Complete characterizations. SIAM J. Optim. 20, 3384-3407 (2010)

[17] Jeyakumar, V., Li, G., Wang, J.H.: Some robust convex programs without a duality gap. J. Convex Anal. 20, 377 - 394 (2013)

[18] Li, G., Ng. K.F.: On extension of Fenchel duality and its application. SIAM J. Optim. 19, 1489-1509 (2008)

[19] Rudin, W.: Functional Analysis (2nd Edition). McGraw-Hill, N.Y. (1991)

[20] Tanino, T.: Conjugate duality in vector optimization. J. Math. Anal. Appl. 167, 84-97 (1992) 